SELECCIONES MATEMÁTICAS
Universidad Nacional de Trujillo
ISSN: 2411-1783 (Online)
Vol. 05(02): $137-153(2018)$

\title{
Transversal Intersection Curves of Two Surfaces in Minkowski 3-Space
}

\section{Curvas dadas por la intersección transversal de dos superficies en el espacio tridimesional de Minkowski}

\author{
Osmar Aléssio*, Sayed A.-N. Badr ${ }^{\dagger}$, Soad A. Hassan, Luciana A. Rodrigues ${ }^{\S}$, Fábio N. \\ Silva I, and M. A. Soliman $\|$
}

Received, Aug. 21, 2018

Accepted, Dec. 03, 2018

DOI: http://dx.doi.org/10.17268/sel.mat.2018.02.02

\begin{abstract}
In this paper, we study the differential geometry of the transversal intersection curve of two surfaces in Minkowski 3-space, where each pair satisfies the following types spacelike-lightlike, timelike-lightlike and lightlike-lightlike. Surfaces are generally give by their parametric or implicit equations, then the surfacesurface intersection problem appear commonly as parametric-parametric, parametric-implicit and implicitimplicit. We derive the Frenet frame, Darboux frame, curvature, torsion, normal curvature and geodesic curvatures of transversal intersections for all types of intersection problems. We show the intersection curve may be spacelike (timelike, lightlike or pseudo null) curve. Finally, we show our methods by given several examples.
\end{abstract}

Keywords. Minkowski 3-Space; Surface-Surface intersection; Pseudo null curve; Null curve; Null frame; Lightlike surface; Darboux Frame.

\section{Resumen}

En este artículo, estudiamos la geometría diferencial de la curva dada por la intersección transversal de dos superficies en el espacio tridimensional de Minkowski donde cada par satisface los siguientes tipos de superficies; tipo espacio - tipo luz, tipo tiempo - tipo luz y tipo luz - tipo luz. Generalmente, las superficies están dadas por sus ecuaciones paramétricas o implícitas, entonces el problema de intersección superficiesuperficie aparece comunmente como paramétrico-paramétrico, paramétrico-implícito e implícito-implícito. Obtenemos el Referencial de Frenet, el Referencial de Darboux, la curvatura, la torsión, la curvatura normal y las curvaturas geodésicas de las intersecciones transversales para todos los tipos de problemas de intersección. Mostramos que la curva de intersección puede ser una curva similar a una curva tipo espacio ( tipo tiempo, tipo luz o pseudo nula). Finalmente, mostramos nuestros métodos por varios ejemplos.

Palabras clave. Espacio tridimensional de Minkowski; Intersección Superficie-superficie; Curva pseudo nula; Curva nula; Referencial nulo; Superficie tipo luz; Referencial de Darboux.

1. Introduction. Lorentz geometry plays an important role in the transition between modern differential geometry and the mathematical physics of general relativity by giving an invariant treatment of Lorentz geometry. The fact that relativity theory is expressed in terms of Lorentz geometry is lucky for geometers, who can thus penetrate surprisingly quickly into cosmology (redshift, expanding universe, and big bang)

\footnotetext{
*Instituto de Ciências Exatas, Naturais e Educação - UFTM, Uberaba, MG, Brazil (osmar. alessio@uftm. edu . br).

$\dagger$ Dept. of Math. Al-Qunfudah University College - Umm Al-Qura University, Al-Qunfudah, KSA (sayed_badr@ymail . com).

${ }^{\ddagger}$ Dept. of Math. Al-Qunfudah University College - Umm Al-Qura University, Al-Qunfudah, KSA (sa odali@ymail . com).

$\S$ Departamento de Matemática - Universidade de Brasília, Brasília, DF, Brazil (luavila@unb.br).

T Centro das Ciências Exatas e das Tecnologias - UFOB, Barreiras, BA, Brazil (fabionuness@ufob.edu.br).

$\|$ Dept. of Math. Faculty of Science - Assiut University, Assiut, Egypt (m_soliman75@yahoo.com). This work is licensed under the Creative Commons Attribution-NoComercial-ShareAlike 4.0.
} 
and, a topic no less interesting geometrically, the gravitation of a single star (perihelion precession, bending of light, and black holes) [19]. There exist four types of curves; spacelike, timelike, pseudo null, and null curves depending on their causal characters. However, the fact that the induced metric on a null curve to be degenerate leads to a much more complicated study and also different from the non degenerate case. In this geometry of null curves difficulties arise because the arc length vanishes, so it is not possible to normalize the tangent vector in the usual way. A method of proceeding is to introduce a new parameter called the pseudo-arc which normalizes the derivative of tangent vector. The primary difference between the lightlike surface and the non-degenerate is that in the first case the normal vector of surface can intersect the tangent space, in other words, a vector of the tangent space $T_{x} M$ can not be uniquely decomposed into a component tangent to $T_{x} M$ and a component of normal space $T_{x} M^{\perp}$. As a result, the lightlike geometry is quite different from Riemannian and semi-Riemannian geometry. From the point of view of physics, null curves and lightlike surfaces are important because they are models of different types and horizons studied in relativity theory. The intersection of two surfaces can be spacelike, timelike and null curves.

The geometric properties of curves and surfaces in the classical literature on differential geometry in Euclidean 3-space $\mathbb{R}^{3}$, can be found in [11]. There is no textbook with a systematic study of curves and surfaces in Minkowski spaces such as it occurs in the Euclidean spaces. A general reference including many topics in semi-Riemannian geometry is the classical book [19]. Differential geometry of the intersection curves in $\mathbb{R}^{3}$ and $\mathbb{R}^{4}$ can be found in $[14,26,23,1,15,7,2,4,8,5,3]$.

Some results on differential geometry of the intersection curves for the transversal intersections in Minkowski 3-space $\mathbb{R}_{1}^{3}$, and Minkowski 4-space $\mathbb{R}_{1}^{4}$, can be found in $[6,22,16]$ and [13] respectively. Aléssio and Guadalupe [6] studied the transversal intersection curve of two parametric spacelike surfaces in $\mathbb{R}_{1}^{3}$ considering parametric-parametric intersection problem. Zafer and Yusuf [22] studied intersection curve of two parametric timelike surfaces in $\mathbb{R}_{1}^{3}$. Karaahmetoglu and Aydemir [16] studied intersection curve of two parametric spacelike and timelike surfaces in $\mathbb{R}_{1}^{3}$. Düldül and Çalışkan [13] compute the Frenet vectors and the curvatures of the spacelike intersection curve of three spacelike hypersurfaces given by their parametric equations in 4-dimensional Minkowski space $\mathbb{R}_{1}^{4}$.

Bonnor [10] described the geometry of null curves in a Minkowski space-time and he proved the fundamental existence and congruence theorems. He introduced the Cartan frame as the most useful one and he uses this frame to study the behaviors of a null curve. Bejancu [9] gave a method for general study of the geometry to null curves in Lorentz manifolds and, more generally, in semi-Riemannian manifolds. Some notations, definitions and reviews of some aspects of the differential geometry in $\mathbb{R}_{1}^{3}$, can be found in $[19,17,18,20,21,25,12,24]$.

This paper presents algorithms for computing the null frame of a given null or pseudo null curve in Minkowski 3-space $\mathbb{R}_{1}^{3}$. Also, it presents the Darboux frame for a spacelike (timelike or null) curve lies on a spacelike (timelike or lightlike) surface. Moreover, it presents algorithms for computing all the differential geometry properties of the transversal intersection curves of two surfaces (spacelike, timelike and lightlike) in Minkowski 3-space $\mathbb{R}_{1}^{3}$. Surfaces are generally given by their parametric or implicit equations, then the surface-surface intersection problem appears commonly as parametric-parametric, parametric-implicit and implicit-implicit. We derive the Frenet frame, Darboux frame, curvature, torsion, normal curvature and geodesic curvatures for transversal intersections for all types of the intersection problems. The intersection curve may be spacelike (timelike, null or pseudo null) curve. We obtain the transversal intersection case at a point, when the normal vector fields are linearly independent.

2. Preliminaries. Minkowski 3-space, $\mathbb{R}_{1}^{3}=\left(\mathbb{R}^{3},\langle,\rangle_{1}\right)$ is a three dimensional real vector space equipped with a Lorentz metric of signature $(2,1)$. For the two vectors $\mathbf{u}=\left(x_{1}, x_{2}, x_{3}\right), \mathbf{v}=\left(y_{1}, y_{2}, y_{3}\right) \in$ $\mathbb{R}_{1}^{3}$, the metric is given by

$$
\langle\mathbf{u}, \mathbf{v}\rangle_{1}=-x_{1} y_{1}+x_{2} y_{2}+x_{3} y_{3} .
$$

A vector $\mathbf{u} \neq \mathbf{0}$ is called spacelike, timelike or lightlike(null), if $\langle\mathbf{u}, \mathbf{u}\rangle_{1}>0,\langle\mathbf{u}, \mathbf{u}\rangle_{1}<0$ or $\langle\mathbf{u}, \mathbf{u}\rangle_{1}=0$, respectively. In particular the vector $\mathbf{u}=\mathbf{0}$ is called spacelike. The norm of the vector $\mathbf{u}$ is defined by,

$$
\|\mathbf{u}\|_{1}=\left|\langle\mathbf{u}, \mathbf{u}\rangle_{1}\right|^{\frac{1}{2}}=\sqrt[2]{\left|-x_{1} x_{1}+x_{2} x_{2}+x_{3} x_{3}\right|} .
$$

The vector $\mathbf{u}$ is called a unit vector if $\langle\mathbf{u}, \mathbf{u}\rangle_{1}= \pm 1$. The vectors $\mathbf{u}$ and $\mathbf{v}$ are said to be orthogonal $(\mathbf{u} \perp \mathbf{v})$, if $\langle\mathbf{u}, \mathbf{v}\rangle_{1}=0$. Similarly, two subsets $U$ and $W$ of $\mathbb{R}_{1}^{3}$ are said to be orthogonal $(U \perp W)$, if $\mathbf{u} \perp \mathbf{w}$ for any $\mathbf{u} \in U$ and $\mathbf{w} \in W$. Denote $W=U^{\perp}=\left\{\mathbf{w} \in \mathbb{R}_{1}^{3}:\langle\mathbf{w}, \mathbf{u}\rangle_{1}=0, \forall \mathbf{u} \in U\right\}$. The vector product of $\mathbf{u}$ and $\mathbf{v}$ (in that order) is the unique vector $\mathbf{u} \times_{1} \mathbf{v} \in \mathbb{R}_{1}^{3}$, where

$$
\mathbf{u} \times_{1} \mathbf{v}=-\mathbf{v} \times_{1} \mathbf{u}=\left|\begin{array}{ccc}
-\mathbf{e}_{1} & \mathbf{e}_{2} & \mathbf{e}_{3} \\
x_{1} & x_{2} & x_{3} \\
y_{1} & y_{2} & y_{3}
\end{array}\right|=\left(x_{3} y_{2}-x_{2} y_{3}, x_{3} y_{1}-x_{1} y_{3}, x_{1} y_{2}-x_{2} y_{1}\right),
$$


where $\left\{\mathbf{e}_{1}, \mathbf{e}_{2}, \mathbf{e}_{3}\right\}$ is the canonical basis of $\mathbb{R}_{1}^{3}$, which satisfy $\mathbf{e}_{1} \times_{1} \mathbf{e}_{2}=-\mathbf{e}_{3}, \mathbf{e}_{2} \times_{1} \mathbf{e}_{3}=\mathbf{e}_{1}$ and $\mathbf{e}_{3} \times_{1} \mathbf{e}_{1}=-\mathbf{e}_{2}$.

Proposition 1. [18] Let $\mathbb{R}_{1}^{3}=\left(\mathbb{R}^{3},\langle,\rangle_{1}\right)$, then

- Two null (lightlike) vectors are linearly dependent if and only if they are orthogonal.

- Two timelike vectors are never orthogonal.

- A timelike vector is never orthogonal to a null (lightlike) vector.

Proposition 2. [18] For $\mathbf{u}, \mathbf{v}, \mathbf{w} \in \mathbb{R}_{1}^{3}$ we have

- $\left\langle\mathbf{u} \times_{1} \mathbf{v}, \mathbf{u}\right\rangle_{1}=0$ and $\left\langle\mathbf{u} \times_{1} \mathbf{v}, \mathbf{v}\right\rangle_{1}=0$.

- $\left\langle\mathbf{u} \times_{1} \mathbf{v}, \mathbf{u} \times_{1} \mathbf{v}\right\rangle_{1}=\langle\mathbf{u}, \mathbf{v}\rangle_{1}^{2}-\langle\mathbf{u}, \mathbf{u}\rangle_{1}\langle\mathbf{v}, \mathbf{v}\rangle_{1}$.

- Let $\mathbf{u}$ be a spacelike vector, $\mathbf{v}$ be a null vector, then $\langle\mathbf{u}, \mathbf{v}\rangle_{1} \neq 0$ if and only if $\mathbf{u} \times_{1} \mathbf{v}$ is spacelike. Also $\langle\mathbf{u}, \mathbf{v}\rangle_{1}=0$ if and only if $\mathbf{u} \times_{1} \mathbf{v}$ is null.

- If $\mathbf{u}$ and $\mathbf{v}$ are null vectors, then $\mathbf{u} \times_{1} \mathbf{v}$ is a spacelike vector.

- If $\mathbf{u}$ is a timelike vector, $\mathbf{v}$ is a null vector, then $\mathbf{u} \times_{1} \mathbf{v}$ is spacelike vector.

Definition 1. A basis $\left\{\mathbf{u}_{1}, \mathbf{u}_{2}, \mathbf{u}_{3}\right\}$ of $\mathbb{R}_{1}^{3}$ is orthonormal if $\left\langle\mathbf{u}_{i}, \mathbf{u}_{j}\right\rangle_{1}=\delta_{i j}$, where

$$
\delta_{i j}=\left\{\begin{array}{cll} 
\pm 1 & \text { if } & i=j \\
0 & \text { if } & i \neq j
\end{array}\right.
$$

If $\mathbf{u}_{1}$ and $\mathbf{u}_{2}$ are non-null vectors, then $B=\left\{\mathbf{u}_{1}, \mathbf{u}_{2}, \mathbf{u}_{1} \times_{1} \mathbf{u}_{2}\right\}$ is a basis of $\mathbb{R}_{1}^{3}$. However, in contrast to the Euclidean space, the casual character of $\mathbf{u}_{1}$ and $\mathbf{u}_{2}$ determines if the basis is or is not positively oriented. Exactly, if $\mathbf{u}_{1}, \mathbf{u}_{2}$ are spacelike, then $\mathbf{u}_{1} \times_{1} \mathbf{u}_{2}$ is timelike and $B$ is negatively oriented because $\operatorname{det}\left(\mathbf{u}_{1}, \mathbf{u}_{2}, \mathbf{u}_{1} \times_{1} \mathbf{u}_{2}\right)=\left\langle\mathbf{u}_{1} \times_{1} \mathbf{u}_{2}, \mathbf{u}_{1} \times_{1} \mathbf{u}_{2}\right\rangle_{1}<0$. If $\mathbf{u}_{1}$ and $\mathbf{u}_{2}$ have different causal character, then $B$ is positively oriented.

Given $U \subset \mathbb{R}_{1}^{3}$ a vector subspace, we consider the induced metric $<\mathbf{u}, \mathbf{v}>_{U}=<\mathbf{u}, \mathbf{v}>_{1}$ such that $\mathbf{u}, \mathbf{v} \in U$. If the metric in $U$ is positive definite it is called spacelike subspace, if the metric in $U$ has index 1 it is called timelike subspace and if the metric in $U$ is degenerate it is called lightlike subspace .

Proposition 3. [18] Let $P \subset \mathbb{R}_{1}^{3}$ be a vector plane. Denote by $\mathbf{N}$ an orthogonal vector with respect to the Euclidean metric, then $P$ is a spacelike (resp. timelike, lightlike) plane if and only if $\mathbf{N}$ is a timelike (resp. spacelike, lightlike) vector.

3. Differential Geometry of Curves in $\mathbb{R}_{1}^{3}$. A regular curve $\alpha(s): I \subset \mathbb{R} \rightarrow \mathbb{R}_{1}^{3}$ can be locally spacelike, timelike or null (lightlike), if its velocity vector field $\left(\alpha^{\prime}(s)\right)$ is spacelike, timelike or null (lightlike), respectively. In particular, if $\alpha^{\prime}(s)$ is spacelike and the acceleration vector field $\left(\alpha^{\prime \prime}(s)\right)$ is null, in this case the curve is called pseudo null curve. The notations for differentiation of the curve $\alpha(s)$ with respect to the arc length $(s)$ are $\alpha^{\prime}(s)=\frac{d \alpha}{d s}, \alpha^{\prime \prime}(s)=\frac{d^{2} \alpha}{d s^{2}}, \alpha^{\prime \prime \prime}(s)=\frac{d^{3} \alpha}{d s^{3}}$ and $\alpha^{(n)}(s)=\frac{d^{n} \alpha}{d s^{n}}, \quad(n>3)$. A non-null curve $\alpha(s)$ is said to be parametrized by arc length parameter $s$, if $\left\langle\alpha^{\prime}(s), \alpha^{\prime}(s)\right\rangle_{1}= \pm 1$. In this case, the curve is called a unit speed curve. A non-null curve $\alpha(s)$ is a straight-line, if $\alpha^{\prime \prime}(s)=\mathbf{0}$. A null (lightlike) curve $\alpha(s)$ is said to be pseudo-parametrized by arc length (or distinguished) if $\alpha(s)$ is not a straight-line and $\left\langle\alpha^{\prime \prime}(s), \alpha^{\prime \prime}(s)\right\rangle_{1}=1$. A null (lightlike) curve $\alpha(s)$, is a straight-line if $\left\langle\alpha^{\prime \prime}(s), \alpha^{\prime \prime}(s)\right\rangle_{1}=0$.

Definition 2. [12] The null frame $F=\{\mathbf{t}, \mathbf{n}, \mathbf{b}\}$ at a point of the null (or pseudo null) curve in $\mathbb{R}_{1}^{3}$ is a positively oriented 3-tuple of vectors satisfying, respectively:

$$
\left\{\begin{array}{l}
\langle\mathbf{t}, \mathbf{t}\rangle_{1}=\langle\mathbf{b}, \mathbf{b}\rangle_{1}=\langle\mathbf{t}, \mathbf{n}\rangle_{1}=\langle\mathbf{n}, \mathbf{b}\rangle_{1}=0,\langle\mathbf{n}, \mathbf{n}\rangle_{1}=\langle\mathbf{t}, \mathbf{b}\rangle_{1}=1, \mathbf{t} \times_{1} \mathbf{b}=\mathbf{n}, \mathbf{n} \times_{1} \mathbf{t}=\mathbf{t}, \\
\mathbf{b} \times_{1} \mathbf{n}=\mathbf{b}, \text { where } \mathbf{b}=\frac{1}{\langle\mathbf{u}, \mathbf{t}\rangle_{1}}\left\{\mathbf{u}-\frac{\langle\mathbf{u}, \mathbf{u}\rangle_{1}}{2\langle\mathbf{u}, \mathbf{t}\rangle_{1}} \mathbf{t}\right\}, \mathbf{u} \in T_{\alpha(s)} M,\langle\mathbf{u}, \mathbf{n}\rangle_{1}=0 \text { and }\langle\mathbf{u}, \mathbf{t}\rangle_{1} \neq 0,
\end{array}\right.
$$

or

$$
\left\{\begin{array}{l}
\langle\mathbf{n}, \mathbf{n}\rangle_{1}=\langle\mathbf{b}, \mathbf{b}\rangle_{1}=\langle\mathbf{t}, \mathbf{n}\rangle_{1}=\langle\mathbf{t}, \mathbf{b}\rangle_{1}=0,\langle\mathbf{t}, \mathbf{t}\rangle_{1}=\langle\mathbf{n}, \mathbf{b}\rangle_{1}=1, \mathbf{t} \times_{1} \mathbf{n}=\mathbf{n}, \mathbf{n} \times_{1} \mathbf{b}=\mathbf{t}, \\
\mathbf{b} \times_{1} \mathbf{t}=\mathbf{b}, \text { where } \mathbf{b}=\frac{1}{\langle\mathbf{u}, \mathbf{n}\rangle_{1}}\left\{\mathbf{u}-\frac{\langle\mathbf{u}, \mathbf{u}\rangle_{1}}{2\langle\mathbf{u}, \mathbf{n}\rangle_{1}} \mathbf{n}\right\}, \mathbf{u} \in T_{\alpha(s)} M,\langle\mathbf{u}, \mathbf{n}\rangle_{1} \neq 0 \text { and }\langle\mathbf{u}, \mathbf{t}\rangle_{1}=0 .
\end{array}\right.
$$

3.1. Frenet Frame. The Frenet frame $\{\mathbf{t}, \mathbf{n}, \mathbf{b}\}$ is a basis of $\mathbb{R}_{1}^{3}$ whose assign for each point of a regular curve $\alpha(s)$, whose variation describes the geometry of the curve. In Euclidean space, the Frenet frame is a positive oriented orthonormal basis, with $\mathbf{b}=\mathbf{t} \times_{\mathbf{1}} \mathbf{n}$. However it has some differences in Minkowski 3-space. 
3.1.1. Spacelike and Timelike Curves. The Frenet frame $\{\mathbf{t}, \mathbf{n}, \mathbf{b}\}$ of a spacelike (or a timelike) regular curve with arc length parametrization, $\alpha(s)$ and $\left\langle\alpha^{\prime \prime}(s), \alpha^{\prime \prime}(s)\right\rangle_{1} \neq 0$ is given by

$$
\mathbf{t}=\alpha^{\prime}(s) \quad \mathbf{n}=\frac{\alpha^{\prime \prime}(s)}{\left\|\alpha^{\prime \prime}(s)\right\|_{1}} \quad \mathbf{b}=\mathbf{t} \times_{1} \mathbf{n}=\frac{\alpha^{\prime}(s) \times_{1} \alpha^{\prime \prime}(s)}{\left\|\alpha^{\prime}(s) \times_{1} \alpha^{\prime \prime}(s)\right\|_{1}} .
$$

The curvature $\kappa(s)$ and the torsion $\tau(s)$, are given as follows

$$
\kappa(s)=\left\|\alpha^{\prime \prime}(s)\right\|_{1}, \quad \tau(s)=-\epsilon_{2} \frac{\left\langle\alpha^{\prime \prime \prime}(s), \mathbf{b}\right\rangle_{1}}{\left\|\alpha^{\prime \prime}(s)\right\|_{1}} .
$$

The Frenet formulas are given as follows

$$
\left[\begin{array}{c}
\mathbf{t}^{\prime} \\
\mathbf{n}^{\prime} \\
\mathbf{b}^{\prime}
\end{array}\right]=\left[\begin{array}{ccc}
0 & \epsilon_{2} \kappa & 0 \\
-\epsilon_{1} \kappa & 0 & -\epsilon_{3} \tau \\
0 & \epsilon_{2} \tau & 0
\end{array}\right]\left[\begin{array}{l}
\mathbf{t} \\
\mathbf{n} \\
\mathbf{b}
\end{array}\right]
$$

where $\epsilon_{1}=\langle\mathbf{t}, \mathbf{t}\rangle_{1}, \epsilon_{2}=\langle\mathbf{n}, \mathbf{n}\rangle_{1}, \epsilon_{3}=\langle\mathbf{b}, \mathbf{b}\rangle_{1}$.

Let $\beta: J \subset \mathbb{R} \rightarrow \mathbb{R}_{1}^{3}$ be a regular curve parametrized by parameter $u$, with the same trace of the curve $\alpha(s)$, i.e. $\beta(u)=\alpha(s(u)), s(u)=\int_{u_{0}}^{u} \sqrt{\epsilon_{1}\left\langle\frac{d \beta(\tau)}{d u}, \frac{d \beta(\tau)}{d u}\right\rangle_{1}} d \tau$ and $\frac{d s}{d u}=\epsilon_{1}\left\langle\frac{d \beta(u)}{d u}, \frac{d \beta(u)}{d u}\right\rangle_{1}$. Thus,

$$
\mathbf{t}=\frac{d \alpha}{d s}=\frac{\frac{d \beta}{d u}}{\frac{d s}{d u}}, \quad \mathbf{n}=\frac{d^{2} \alpha}{d s^{2}}=\frac{\frac{d^{2} \beta}{d u^{2}}-\frac{d^{2} s}{d u^{2}} \mathbf{t}}{\left(\frac{d s}{d u}\right)^{2}}, \quad \frac{d^{3} \alpha}{d s^{3}}=\frac{\frac{d^{3} \beta}{d u^{3}}-3 \frac{d s}{d u} \frac{d^{2} s}{d u^{2}} \mathbf{n}+\frac{d^{3} s}{d u^{3}} \mathbf{t}}{\left(\frac{d s}{d u}\right)^{3}} .
$$

3.1.2. Pseudo Null Curves. Let $\alpha(s)=(x(s), y(s), z(s))$ be a regular pseudo null with unit speed curve, such that $\alpha^{\prime}(s), \alpha^{\prime \prime}(s), \alpha^{\prime \prime \prime}(s)$ are different from zero for all $s$, then there exists only one Frenet frame (null frame) $\{\mathbf{t}, \mathbf{n}, \mathbf{b}\}$ have the same orientations with $\left\{\alpha^{\prime}(s), \alpha^{\prime \prime}(s), \alpha^{\prime \prime \prime}(s)\right\}$, such that $\langle\mathbf{n}, \mathbf{n}\rangle_{1}=$ $\langle\mathbf{b}, \mathbf{b}\rangle_{1}=\langle\mathbf{t}, \mathbf{n}\rangle_{1}=\langle\mathbf{t}, \mathbf{b}\rangle_{1}=0,\langle\mathbf{t}, \mathbf{t}\rangle_{1}=\langle\mathbf{n}, \mathbf{b}\rangle_{1}=1, \mathbf{t} \times_{1} \mathbf{n}=\mathbf{n}, \mathbf{n} \times_{1} \mathbf{b}=\mathbf{t}, \mathbf{b} \times_{1} \mathbf{t}=\mathbf{b}$. This frame is a pseudo-orthonormal basis in $\mathbb{R}_{1}^{3}$ and it can be constructed as the following,

Let $\mathbf{t}=\alpha^{\prime}(s)=\left(x^{\prime}(s), y^{\prime}(s), z^{\prime}(s)\right), \mathbf{n}=\alpha^{\prime \prime}(s)=\left(x^{\prime \prime}(s), y^{\prime \prime}(s), z^{\prime \prime}(s)\right)$ and $\mathbf{V}=(1, u, v)$, such that

$$
\langle\mathbf{t}, \mathbf{t}\rangle_{1}=1,\langle\mathbf{n}, \mathbf{n}\rangle_{1}=0,\langle\mathbf{t}, \mathbf{n}\rangle_{1}=0,\langle\mathbf{V}, \mathbf{t}\rangle_{1}=0,\langle\mathbf{V}, \mathbf{n}\rangle_{1} \neq 0 \text {. }
$$

Thus, we have

$$
\begin{array}{cl}
x^{2}=y^{\prime 2}+z^{\prime 2}-1, & x^{\prime \prime 2}=y^{\prime \prime 2}+z^{\prime \prime^{2}}, \\
x^{\prime}=u y^{\prime}+v z^{\prime}, & x^{\prime \prime} \neq u y^{\prime \prime}+v z^{\prime \prime} .
\end{array}
$$

Since $\mathbf{n}$ is a null vector, then $x^{\prime \prime} \neq 0$, then we can put

$$
u y^{\prime \prime}+v z^{\prime \prime}=0 .
$$

Solving (3.7) and (3.8) for the $u$, get

$$
u=\frac{x^{\prime} z^{\prime \prime}}{y^{\prime} z^{\prime \prime}-z^{\prime} y^{\prime \prime}}, \quad v=\frac{-x^{\prime} y^{\prime \prime}}{y^{\prime} z^{\prime \prime}-z^{\prime} y^{\prime \prime}}
$$

Solving (3.7) for $x^{\prime \prime}$, we obtain

$$
x^{\prime \prime}= \pm\left(y^{\prime} z^{\prime \prime}-z^{\prime} y^{\prime \prime}\right) .
$$

Thus, the vector $\mathbf{V}$ is given by

$$
\mathbf{V}=\left(1, \frac{x^{\prime} z^{\prime \prime}}{\delta x^{\prime \prime}}, \frac{-x^{\prime} y^{\prime \prime}}{\delta x^{\prime \prime}}\right) ; \quad \delta=\frac{\left(y^{\prime} z^{\prime \prime}-z^{\prime} y^{\prime \prime}\right)}{x^{\prime \prime}}
$$

Using (3.2), we get

$$
\mathbf{b}=-\frac{\delta}{2 x^{\prime \prime 2}}\left(\left(2 \delta x^{\prime \prime}, 2 x^{\prime} z^{\prime \prime},-2 x^{\prime} y^{\prime \prime}\right)+\delta\left(x^{2}-1\right) \alpha^{\prime \prime}\right) ; \quad \delta=\frac{\left(y^{\prime} z^{\prime \prime}-z^{\prime} y^{\prime \prime}\right)}{x^{\prime \prime}},
$$

and the null frame is given by

$$
\mathbf{t}=\alpha^{\prime}, \quad \mathbf{n}=\alpha^{\prime \prime}, \quad \mathbf{b}=-\frac{\delta}{2 x^{\prime \prime 2}}\left[\left(2 \delta x^{\prime \prime}, 2 x^{\prime} z^{\prime \prime},-2 x^{\prime} y^{\prime \prime}\right)+\delta\left(x^{\prime 2}-1\right) \mathbf{n}\right],
$$


where

$$
\delta=\frac{\left(y^{\prime} z^{\prime \prime}-z^{\prime} y^{\prime \prime}\right)}{x^{\prime \prime}}
$$

The Frenet formulas are given by

$$
\left[\begin{array}{l}
\mathbf{t}^{\prime} \\
\mathbf{n}^{\prime} \\
\mathbf{b}^{\prime}
\end{array}\right]=\left[\begin{array}{ccc}
0 & 1 & 0 \\
0 & -\tau & 0 \\
-1 & 0 & \tau
\end{array}\right]\left[\begin{array}{l}
\mathbf{t} \\
\mathbf{n} \\
\mathbf{b}
\end{array}\right]
$$

In this case the function $\tau$ is called pseudo-torsion along the curve $\alpha(s)$ which is given by

$$
\tau(s)=-\left\langle\alpha^{\prime \prime \prime}, \mathbf{b}\right\rangle_{1}
$$

Let $\beta: J \subset \mathbb{R} \rightarrow \mathbb{R}_{1}^{3}$ be a regular curve parametrized by parameter $u$, with the same trace of the pseudo null curve $\alpha(s)$, i.e. $\beta(u)=\alpha(s(u)), s(u)=\int_{u_{0}}^{u} \sqrt{\left\langle\frac{d \beta(\tau)}{d u}, \frac{d \beta(\tau)}{d u}\right\rangle_{1}} d \tau$ and $\frac{d s}{d u}=\left\langle\frac{d \beta(u)}{d u}, \frac{d \beta(u)}{d u}\right\rangle_{1}$. Thus,

$$
\mathbf{t}=\frac{d \alpha}{d s}=\frac{\frac{d \beta}{d u}}{\frac{d s}{d u}}, \quad \mathbf{n}=\frac{d^{2} \alpha}{d s^{2}}=\frac{\frac{d^{2} \beta}{d u^{2}}-\frac{d^{2} s}{d u^{2}} \mathbf{t}}{\left(\frac{d s}{d u}\right)^{2}}, \quad \frac{d^{3} \alpha}{d s^{3}}=\frac{\frac{d^{3} \beta}{d u^{3}}-3 \frac{d s}{d u} \frac{d^{2} s}{d u^{2}} \mathbf{n}+\frac{d^{3} s}{d u^{3}} \mathbf{t}}{\left(\frac{d s}{d u}\right)^{3}} .
$$

3.1.3. Null curves. Let $\alpha(s)$ be a null curve in $\mathbb{R}_{1}^{3}$ parametrized with pseudo arc length $s$ and $\alpha^{\prime}(s)$, $\alpha^{\prime \prime}(s), \alpha^{\prime \prime \prime}(s)$ are linearly independent for all $s$, then there exists only one Frenet frame (null frame), $\{\mathbf{n}, \mathbf{t}, \mathbf{b}\}$ have the same orientations with $\left\{\alpha^{\prime}(s), \alpha^{\prime \prime}(s), \alpha^{\prime \prime \prime}(s)\right\}$, such that, $\langle\mathbf{t}, \mathbf{t}\rangle_{1}=\langle\mathbf{b}, \mathbf{b}\rangle_{1}=\langle\mathbf{t}, \mathbf{n}\rangle_{1}=$ $\langle\mathbf{n}, \mathbf{b}\rangle_{1}=0,\langle\mathbf{n}, \mathbf{n}\rangle_{1}=\langle\mathbf{t}, \mathbf{b}\rangle_{1}=1, \mathbf{t} \times_{1} \mathbf{b}=\mathbf{n}, \mathbf{n} \times_{1} \mathbf{t}=\mathbf{t}, \mathbf{b} \times_{1} \mathbf{n}=\mathbf{b}$. This frame is a pseudoorthonormal basis in $\mathbb{R}_{1}^{3}$ and it can be constructed as previous section 3.1.2, then the null frame is given by

$$
\mathbf{t}=\alpha^{\prime}, \quad \mathbf{n}=\alpha^{\prime \prime}, \quad \mathbf{b}=-\frac{\sigma}{2 x^{\prime 2}}\left(\left(2 \sigma x^{\prime}, 2 x^{\prime \prime} z^{\prime},-2 x^{\prime \prime} y^{\prime}\right)+\sigma\left(x^{\prime \prime 2}-1\right) \mathbf{t}\right),
$$

where

$$
\sigma=\frac{\left(y^{\prime \prime} z^{\prime}-z^{\prime \prime} y^{\prime}\right)}{x^{\prime}}
$$

The Frenet formulas are given by

$$
\left[\begin{array}{c}
\mathbf{n}^{\prime} \\
\mathbf{t}^{\prime} \\
\mathbf{b}^{\prime}
\end{array}\right]=\left[\begin{array}{ccc}
0 & -\tau & -1 \\
1 & 0 & 0 \\
\tau & 0 & 0
\end{array}\right]\left[\begin{array}{c}
\mathbf{n} \\
\mathbf{t} \\
\mathbf{b}
\end{array}\right]
$$

In this case, the function $\tau$ is called pseudo-torsion along the curve $\alpha(s)$ which is given by

$$
\tau(s)=-\left\langle\alpha^{\prime \prime \prime}, \mathbf{b}\right\rangle_{1} .
$$

Let $\beta: J \subset \mathbb{R} \rightarrow \mathbb{R}_{1}^{3}$ be a regular curve parametrized by parameter $u$, with the same trace of the null curve $\alpha(s)$, i.e. $\beta(u)=\alpha(s(u)), s(u)=\int_{u_{0}}^{u} \sqrt{\left\langle\frac{d^{2} \beta(\tau)}{d u^{2}}, \frac{d^{2} \beta(\tau)}{d u^{2}}\right\rangle_{1}} d \tau$ and $\frac{d s}{d u}=\left\langle\frac{d^{2} \beta(u)}{d u^{2}}, \frac{d^{2} \beta(u)}{d u^{2}}\right\rangle_{1}$. Thus,

$$
\mathbf{t}=\frac{d \alpha}{d s}=\frac{\frac{d \beta}{d u}}{\frac{d s}{d u}}, \quad \mathbf{n}=\frac{d^{2} \alpha}{d s^{2}}=\frac{\frac{d^{2} \beta}{d u^{2}}-\frac{d^{2} s}{d u^{2}} \mathbf{t}}{\left(\frac{d s}{d u}\right)^{2}}, \quad \frac{d^{3} \alpha}{d s^{3}}=\frac{\frac{d^{3} \beta}{d u^{3}}-3 \frac{d s}{d u} \frac{d^{2} s}{d u^{2}} \mathbf{n}+\frac{d^{3} s}{d u^{3}} \mathbf{t}}{\left(\frac{d s}{d u}\right)^{3}} .
$$

Notice that, if $\frac{d s}{d u}=0$ then the curve is a straight-line.

4. Curves on Surfaces in $\mathbb{R}_{1}^{3}$. A surface in $\mathbb{R}_{1}^{3}$ is called spacelike, timelike or lightlike, if the induced metric on the surface is positive definite, has index 1 or degenerate respectively. In other words, the surface in $\mathbb{R}_{1}^{3}$ is called spacelike, timelike or lightlike, if the normal vector field on the surface is timelike, spacelike or lightlike. For more details, see [19]. 
4.1. Curves on Parametric Surfaces in $\mathbb{R}_{1}^{3}$. Consider an arbitrary parametric spacelike (timelike or lightlike) surface $\boldsymbol{X}\left(u_{1}, u_{2}\right)$, the vector

$$
\zeta=\boldsymbol{X}_{1} \times \boldsymbol{X}_{2}
$$

(where $\left.\boldsymbol{X}_{r}=\frac{\partial \boldsymbol{X}}{\partial u_{r}}(r=1,2)\right)$ is the normal vector field of the surface $\boldsymbol{X}$. It is regular if $\zeta \neq 0$. The coefficients of the first fundamental form are given by

$$
g_{i j}=\left\langle\boldsymbol{X}_{i}, \boldsymbol{X}_{j}\right\rangle_{1} ; \quad i, j=1,2
$$

Let $u_{r}=u_{r}(u), r=1,2$ be functions in the $u_{1} u_{2}$-plane which defines a regular curve parametrized by an arbitrary parameter $u$ on the surface $\boldsymbol{X}$, as follows

$$
\gamma(u)=\boldsymbol{X}\left(u_{1}(u), u_{2}(u)\right)=\left(x_{1}(u), x_{2}(u), x_{3}(u)\right)
$$

Differentiating (4.2) three times with respect to the parameter $u$, then projecting the vectors $\gamma^{\prime}(u)$ and $\gamma^{\prime \prime}(u), \gamma^{\prime \prime \prime}(u)$ onto the normal vector field $\zeta$ of the surface $\mathbf{X}$, we get

$$
\left\langle\gamma^{\prime}(u), \zeta\right\rangle_{1}=0
$$

$$
\left\langle\gamma^{\prime \prime}(u), \zeta\right\rangle_{1}=\left\langle\mathbf{X}_{11}, \zeta\right\rangle_{1}\left(u_{1}^{\prime}\right)^{2}+2\left\langle\mathbf{X}_{12}, \zeta\right\rangle_{1} u_{1}^{\prime} u_{2}^{\prime}+\left\langle\mathbf{X}_{22}, \zeta\right\rangle_{1}\left(u_{2}^{\prime}\right)^{2}
$$

$$
\begin{aligned}
\left\langle\gamma^{\prime \prime \prime}(u), \zeta\right\rangle_{1}= & \left\langle\boldsymbol{X}_{111}, \zeta\right\rangle_{1}\left(u_{1}^{\prime}\right)^{3}+\left\langle\boldsymbol{X}_{222}, \zeta\right\rangle_{1}\left(u_{2}^{\prime}\right)^{3}+3\left\langle\boldsymbol{X}_{112}, \zeta\right\rangle_{1}\left(u_{1}^{\prime}\right)^{2} u_{2}^{\prime}+3\left\langle\boldsymbol{X}_{122}, \zeta\right\rangle u_{1}^{\prime}\left(u_{2}^{\prime}\right)^{2} \\
& +3\left(\left\langle\mathbf{X}_{11}, \zeta\right\rangle_{1} u_{1}^{\prime}+\left\langle\mathbf{X}_{12}, \zeta\right\rangle_{1} u_{2}^{\prime}\right) u_{1}^{\prime \prime}+3\left(\left\langle\mathbf{X}_{12}, \zeta\right\rangle_{1} u_{1}^{\prime}+\left\langle\mathbf{X}_{22}, \zeta\right\rangle_{1} u_{2}^{\prime}\right) u_{2}^{\prime \prime},
\end{aligned}
$$

where $\boldsymbol{X}_{i j}=\frac{\partial^{2} \boldsymbol{X}}{\partial u_{i} \partial u_{j}}, \boldsymbol{X}_{i j k}=\frac{\partial^{3} \boldsymbol{X}}{\partial u_{i} \partial u_{j} \partial u_{k}}(i, j, k=1,2)$. Projecting the vectors $\gamma^{\prime}(u), \gamma^{\prime \prime}(u)$ and $\gamma^{\prime \prime \prime}(u)$ onto $\mathbf{X}_{1}$ and $\mathbf{X}_{2}$, we obtain

$$
g_{11} u_{1}^{\prime}+g_{12} u_{2}^{\prime}=\left\langle\gamma^{\prime}(u), \mathbf{X}_{1}\right\rangle_{1}, \quad g_{12} u_{1}^{\prime}+g_{22} u_{2}^{\prime}=\left\langle\gamma^{\prime}(u), \mathbf{X}_{2}\right\rangle_{1},
$$

$$
\left\{\begin{array}{l}
g_{11} u_{1}^{\prime \prime}+g_{12} u_{2}^{\prime \prime}=\left\langle\gamma^{\prime \prime}(u), \mathbf{X}_{1}\right\rangle_{1}-\left\langle\mathbf{X}_{11}, \mathbf{X}_{1}\right\rangle_{1}\left(u_{1}^{\prime}\right)^{2}-2\left\langle\mathbf{X}_{12}, \mathbf{X}_{1}\right\rangle_{1} u_{1}^{\prime} u_{2}^{\prime}-\left\langle\mathbf{X}_{22}, \mathbf{X}_{1}\right\rangle_{1}\left(u_{2}^{\prime}\right)^{2} \\
g_{12} u_{1}^{\prime \prime}+g_{22} u_{2}^{\prime \prime}=\left\langle\gamma^{\prime \prime}(u), \mathbf{X}_{2}\right\rangle_{1}-\left\langle\mathbf{X}_{11}, \mathbf{X}_{2}\right\rangle_{1}\left(u_{1}^{\prime}\right)^{2}-2\left\langle\mathbf{X}_{12}, \mathbf{X}_{2}\right\rangle_{1} u_{1}^{\prime} u_{2}^{\prime}-\left\langle\mathbf{X}_{22}, \mathbf{X}_{2}\right\rangle_{1}\left(u_{2}^{\prime}\right)^{2} .
\end{array}\right.
$$

4.1.1. Curves on Parametric Spacelike and Timelike Surfaces in $\mathbb{R}_{1}^{3}$. The unit normal vector field $\mathbf{N}$ of a parametric spacelike (or a timelike) surface $\mathbf{X}$ is given by

$$
\mathbf{N}=\frac{\zeta}{\|\zeta\|_{1}}
$$

Solving (4.5) and using proposition 2, we get

$$
u_{1}^{\prime}=\frac{\left\langle\gamma^{\prime}(u) \times_{1} \mathbf{X}_{2}, \zeta\right\rangle_{1}}{\langle\zeta, \zeta\rangle_{1}} . \quad u_{2}^{\prime}=\frac{\left\langle\mathbf{X}_{1} \times_{1} \gamma^{\prime}(u), \zeta\right\rangle_{1}}{\langle\zeta, \zeta\rangle_{1}}
$$

Solving (4.6) and using proposition 2, we get

$$
\left\{\begin{array}{l}
u_{1}^{\prime \prime}=\frac{\left\langle\gamma^{\prime \prime}(u) \times_{1} \mathbf{X}_{2}, \zeta\right\rangle_{1}-\left\langle\mathbf{X}_{11} \times_{1} \mathbf{X}_{2}, \zeta\right\rangle_{1}\left(u_{1}^{\prime}\right)^{2}-2\left\langle\mathbf{X}_{12} \times_{1} \mathbf{X}_{2}, \zeta\right\rangle_{1} u_{1}^{\prime} u_{2}^{\prime}-\left\langle\mathbf{X}_{22} \times_{1} \mathbf{X}_{2}, \zeta\right\rangle_{1}\left(u_{2}^{\prime}\right)^{2}}{\langle\zeta, \zeta\rangle_{1}} \\
u_{2}^{\prime}=\frac{-\left\langle\gamma^{\prime \prime}(u) \times_{1} \mathbf{X}_{1}, \zeta\right\rangle_{1}+\left\langle\mathbf{X}_{11} \times_{1} \mathbf{X}_{1}, \zeta\right\rangle_{1}\left(u_{1}^{\prime}\right)^{2}+2\left\langle\mathbf{X}_{12} \times_{1} \mathbf{X}_{1}, \zeta\right\rangle_{1} u_{1}^{\prime} u_{2}^{\prime}+\left\langle\mathbf{X}_{22} \times_{1} \mathbf{X}_{1}, \zeta\right\rangle_{1}\left(u_{2}^{\prime}\right)^{2}}{\langle\zeta, \zeta\rangle_{1}}
\end{array}\right.
$$


4.1.2. Curves on Parametric Lightlike Surfaces in $\mathbb{R}_{1}^{3}$. The normal vector field $\zeta$ of a parametric lightlike surface $\mathbf{X}$ is given by (4.1). If $\mathbf{X}_{i}$ is lightlike, $\mathbf{X}_{j}$ is spacelike and $g_{12}=0$, then by using the proposition 2, we obtain

$$
\zeta=\mathbf{X}_{i}
$$

where $\{i, j\}=\{1,2\}$. Thus the normal vector field $\zeta$ of a parametric lightlike surface $\mathbf{X}$ is given by

$$
\zeta=\left\{\begin{array}{ccc}
\mathbf{X}_{i} & \text { if } & \left\|\boldsymbol{X}_{1} \times \boldsymbol{X}_{2}\right\|_{1}=\left\|\mathbf{X}_{i}\right\|_{1}=0, i=1,2, \\
\boldsymbol{X}_{1} \times \boldsymbol{X}_{2} & \text { if } & \left\|\boldsymbol{X}_{1} \times \boldsymbol{X}_{2}\right\|_{1}=0 .
\end{array}\right.
$$

In this case (4.7) will reduced to one equation or vanish. Since the vector $\gamma^{\prime}(u)$ can be written as,

$$
\vartheta^{1} u_{1}^{\prime}+\varsigma^{1} u_{2}^{\prime}=x^{\prime}, \quad \vartheta^{2} u_{1}^{\prime}+\varsigma^{2} u_{2}^{\prime}=y^{\prime}, \quad \vartheta^{3} u_{1}^{\prime}+\varsigma^{3} u_{2}^{\prime}=z^{\prime},
$$

where $\gamma^{\prime}(u)=\left(x^{\prime}, y^{\prime}, z^{\prime}\right), \mathbf{X}_{1}=\left(\vartheta^{1}, \vartheta^{2}, \vartheta^{3}\right)$ and $\mathbf{X}_{2}=\left(\varsigma^{1}, \varsigma^{2}, \varsigma^{3}\right)$, then we can compute $u_{1}^{\prime}$ and $u_{2}^{\prime}$ by solving two suitable equations of (4.7) and (4.10). By the same way we can compute $u_{1}^{\prime \prime}$ and $u_{2}^{\prime \prime}$.

4.2. Curves on Implicit Surfaces in $\mathbb{R}_{1}^{3}$. Consider an arbitrary implicit spacelike (timelike or lightlike) surface

$$
S=\left\{\left(x_{1}, x_{2}, x_{3}\right) ; h\left(x_{1}, x_{2}, x_{3}\right)=0\right\},
$$

where $h\left(x_{1}, x_{2}, x_{3}\right): \mathbb{R}_{1}^{3} \longrightarrow \mathbb{R}$ is a differential function. The vector field

$$
\zeta_{h}=\left(-h_{1}, h_{2}, h_{3}\right) ; \quad h_{i}=\frac{\partial h}{\partial x_{i}}, i=1,2,3,
$$

is orthogonal to any vector of the tangent plane. We assume that the surface is regular, in other words $\zeta_{h} \neq \mathbf{0}$. Let $\gamma(u)$ is a regular curve on the surface $S$, parametrized by an arbitrary parameter $u$ and it is defined by

$$
\gamma(u)=\left(x_{1}(u), x_{2}(u), x_{3}(u)\right), \quad h(\gamma(u))=0
$$

Differentiating (4.12) with respect to the parameter $u$, then projecting the vector $\gamma^{\prime}(u)$ onto the normal vector field $\zeta$ of the surface $S$, we get

$$
\left\langle\zeta_{h}(u), \gamma^{\prime}(u)\right\rangle_{1}=0
$$

where

$$
\zeta_{h}(u)=\left[\begin{array}{lll}
-h_{1}(\gamma(u)) & h_{2}(\gamma(u)) & h_{3}(\gamma(u))
\end{array}\right], \quad \gamma^{\prime}(u)=\left[\begin{array}{lll}
x_{1}^{\prime}(u) & x_{2}^{\prime}(u) & x_{3}^{\prime}(u)
\end{array}\right] .
$$

Differentiation (4.13) with respect to $u$ yields,

$$
\left\langle\zeta_{h}(u), \gamma^{\prime \prime}(u)\right\rangle_{1}=-\left\langle\gamma^{\prime} *\left(H^{h}\right)^{T},\left(\gamma^{\prime}\right)\right\rangle_{1},
$$

where

$$
\gamma^{\prime \prime}(u)=\left[\begin{array}{lll}
x_{1}^{\prime \prime}(u) & x_{2}^{\prime \prime}(u) & x_{3}^{\prime \prime}(u)
\end{array}\right], \quad H^{h}=\left[\begin{array}{ccc}
-h_{11} & -h_{12} & -h_{13} \\
h_{21} & h_{22} & h_{23} \\
h_{31} & h_{32} & h_{33}
\end{array}\right] .
$$

and $*$ denotes to the multiplication of matrices.

Differentiating (4.14) with respect to $u$ we obtain

$$
\left\langle\zeta_{h}(u), \gamma^{\prime \prime \prime}(u)\right\rangle_{1}=-3\left\langle\gamma^{\prime} *\left(H^{h}\right)^{T}, \gamma^{\prime \prime}\right\rangle_{1}-\left\langle\gamma^{\prime} *\left(\frac{d H^{h}}{d u}\right)^{T}, \gamma^{\prime}\right\rangle_{1},
$$

where

$$
\begin{gathered}
\gamma^{\prime \prime \prime}(u)=\left[\begin{array}{lll}
x_{1}^{\prime \prime \prime}(u) & x_{2}^{\prime \prime \prime}(u) & x_{3}^{\prime \prime \prime}(u)
\end{array}\right], \quad\left\langle\gamma^{\prime \prime} *\left(H^{h}\right)^{T}, \gamma^{\prime}\right\rangle_{1}=\left\langle\gamma^{\prime} *\left(H^{h}\right)^{T}, \gamma^{\prime \prime}\right\rangle_{1}, \\
\frac{d H^{h}}{d u}=H_{1}^{h} x_{1}^{\prime}(u)+H_{2}^{h} x_{2}^{\prime}(u)+H_{3}^{h} x_{3}^{\prime}(u) \quad \text { and } H_{i}^{h}=\left[\begin{array}{ccc}
-h_{11 i} & -h_{12 i} & -h_{13 i} \\
h_{12 i} & h_{22 i} & h_{23 i} \\
h_{13 i} & h_{23 i} & h_{33 i}
\end{array}\right] .
\end{gathered}
$$


4.2.1. Curves on Implicit Spacelike and Timelike Surfaces in $\mathbb{R}_{1}^{3}$. The unit normal vector field $\mathbf{N}$ of an implicit spacelike (or a timelike) surface $S$ is given by

$$
\mathbf{N}=\frac{\zeta_{h}}{\|\zeta\|_{1}}
$$

4.2.2. Curves on Implicit Lightlike Surfaces in $\mathbb{R}_{1}^{3}$. The normal vector field $\zeta$ of an implicit lightlike surface $S$ is given by

$$
\zeta=\left(-h_{1}, h_{2}, h_{3}\right)
$$

\subsection{Darboux Frame.}

4.3.1. Darboux Frame of Spacelike, Timelike or Pseudo Null curves on a non lightlike Surfaces. In this subsection we will study the Darboux frame.

Definition 3. If $\{\mathbf{t}, \mathbf{n}, \mathbf{b}\}$ is the Frenet frame of the curve $\alpha(s)$ which lies on a spacelike (timelike) surface $S$ in $\mathbb{R}_{1}^{3}$ with unit normal vector field $\mathbf{N}$, then the orthonormal basis $\{\mathbf{t}, \mathbf{U}, \mathbf{N}\}$, where

$$
\mathbf{U}=\mathbf{N} \times{ }_{1} \mathbf{t}
$$

is called natural frame (Darboux frame) for the curve-surface pair $(\alpha(s), S)$.

Definition 4. [12] The basis $\{\mathbf{t}, \mathbf{U}, \mathbf{N}\}$, where

$$
\left\{\begin{array}{l}
\langle\mathbf{t}, \mathbf{t}\rangle_{1}=\langle\mathbf{U}, \mathbf{U}\rangle_{1}=\langle\mathbf{t}, \mathbf{N}\rangle_{1}=\langle\mathbf{N}, \mathbf{U}\rangle_{1}=0,\langle\mathbf{N}, \mathbf{N}\rangle_{1}=\langle\mathbf{t}, \mathbf{U}\rangle_{1}=1, \mathbf{t} \times_{1} \mathbf{U}=\mathbf{N}, \mathbf{N} \times_{1} \mathbf{t}=\mathbf{t} \\
\mathbf{U} \times_{1} \mathbf{N}=\mathbf{U}, \text { where } \mathbf{U}=\frac{1}{\langle\mathbf{V}, \mathbf{t}\rangle_{1}}\left\{\mathbf{V}-\frac{\langle\mathbf{V}, \mathbf{V}\rangle_{1}}{2\langle\mathbf{V}, \mathbf{t}\rangle_{1}} \mathbf{t}\right\}, \mathbf{V} \in T_{\alpha(s)} M,\langle\mathbf{V}, \mathbf{N}\rangle_{1}=0 \text { and }\langle\mathbf{V}, \mathbf{t}\rangle_{1} \neq 0
\end{array}\right.
$$

or

$\left\{\begin{array}{l}\langle\mathbf{N}, \mathbf{N}\rangle_{1}=\langle\mathbf{U}, \mathbf{U}\rangle_{1}=\langle\mathbf{t}, \mathbf{N}\rangle_{1}=\langle\mathbf{t}, \mathbf{U}\rangle_{1}=0,\langle\mathbf{t}, \mathbf{t}\rangle_{1}=\langle\mathbf{N}, \mathbf{U}\rangle_{1}=1, \mathbf{t} \times{ }_{1} \mathbf{N}=\mathbf{N}, \mathbf{N} \times{ }_{1} \mathbf{U}=\mathbf{t}, \\ \mathbf{U} \times_{1} \mathbf{t}=\mathbf{U}, \text { where } \mathbf{U}=\frac{1}{\langle\mathbf{V}, \mathbf{N}\rangle_{1}}\left\{\mathbf{V}-\frac{\langle\mathbf{V}, \mathbf{V}\rangle_{1}}{2\langle\mathbf{V}, \mathbf{N}\rangle_{1}} \mathbf{N}\right\}, \mathbf{V} \in T_{\alpha(s)} M,\langle\mathbf{V}, \mathbf{N}\rangle_{1} \neq 0 \text { and }\langle\mathbf{v}, \mathbf{t}\rangle_{1}=0,\end{array}\right.$

is called null Darboux frame at a point of the null (or pseudo null) curve in timelike surfaces (or lightlike surfaces), respectively.

Definition 5. [21] Let $S$ be a non lightlike surface in $\mathbb{R}_{1}^{3}$ and $\alpha(s)$ be a curve on $S$, then the functions

$$
\kappa_{g}(s)=\left\langle\mathbf{t}^{\prime}(s), \mathbf{U}(s)\right\rangle_{1}, \quad \kappa_{n}(s)=\left\langle\mathbf{t}^{\prime}(s), \mathbf{N}(s)\right\rangle_{1}, \quad \tau_{g}(s)=\left\langle\mathbf{N}^{\prime}(s), \mathbf{U}(s)\right\rangle_{1},
$$

are called the geodesic curvature, normal curvature and geodesic torsion of the curve $\alpha(s)$, respectively.

Theorem 1. [21, 24] Let $S$ be a non lightlike surface in $\mathbb{R}_{1}^{3}$ and $\alpha(s)$ be a curve on the surface $S$, then the derivative formulas of the natural frame $\{\mathbf{t}, \mathbf{U}, \mathbf{N}\}$, are given by

$$
\left[\begin{array}{c}
\mathbf{t}^{\prime} \\
\mathbf{U}^{\prime} \\
\mathbf{N}^{\prime}
\end{array}\right]=\left[\begin{array}{ccc}
0 & \epsilon_{2}^{*} k_{g} & \epsilon_{3}^{*} k_{n} \\
-\epsilon_{1} k_{g} & 0 & -\epsilon_{3}^{*} \tau_{g} \\
-\epsilon_{1} k_{n} & \epsilon_{2}^{*} \tau_{g} & 0
\end{array}\right]\left[\begin{array}{c}
\mathbf{t} \\
\mathbf{U} \\
\mathbf{N}
\end{array}\right]
$$

where $\epsilon_{1}=\langle\mathbf{t}, \mathbf{t}\rangle_{1}, \epsilon_{2}^{*}=\langle\mathbf{U}, \mathbf{U}\rangle_{1}$ and $\epsilon_{3}^{*}=\langle\mathbf{N}, \mathbf{N}\rangle_{1}$.

4.3.2. Darboux Frame of Spacelike or Pseudo Null curves on Lightlike Surfaces. Let $S$ be a lightlike surface in $\mathbb{R}_{1}^{3}, \alpha(s): I \rightarrow S \subset \mathbb{R}_{1}^{3}$ be a unit speed spacelike curve and it lies on the surface $S, \zeta$ be the normal vector field of the surface $S$, and $\mathbf{t}$ is the unit tangent vector field of the curve $\alpha(s)$, then there exists only one Darboux frame (null frame) $\{\mathbf{t}, \zeta, \mathbf{U}\}$, such that $\langle\zeta, \zeta\rangle_{1}=\langle\mathbf{U}, \mathbf{U}\rangle_{1}=\langle\mathbf{t}, \zeta\rangle_{1}=\langle\mathbf{t}, \mathbf{U}\rangle_{1}=$ $0,\langle\mathbf{t}, \mathbf{t}\rangle_{1}=\langle\zeta, \mathbf{U}\rangle_{1}=1, \mathbf{t} \times{ }_{1} \zeta=\zeta, \zeta \times_{1} \mathbf{U}=\mathbf{t}, \mathbf{U} \times_{1} \mathbf{t}=\mathbf{U}$, and it is a pseudo-orthonormal basis in $\mathbb{R}_{1}^{3}$. This frame satisfies (3.2) and it can be constructed as in section 3.1.2, so it is given by

$$
\mathbf{t}=\left(x^{\prime}, y^{\prime}, z^{\prime}\right), \quad \zeta=\left(\zeta^{1}, \zeta^{2}, \zeta^{3}\right), \quad \mathbf{U}=-\frac{\gamma}{2\left(\zeta^{1}\right)^{2}}\left[\left(2 \gamma \zeta^{1}, 2 x^{\prime} \zeta^{3},-2 x^{\prime} \zeta^{2}\right)+\gamma\left(x^{\prime 2}-1\right) \zeta\right],
$$

where

$$
\gamma=\frac{\left(y^{\prime} \zeta^{3}-z^{\prime} \zeta^{2}\right)}{\zeta^{1}}
$$


Definition 6. Let $S$ be a lightlike surface in $\mathbb{R}_{1}^{3}$ and $\alpha(s)$ be a curve on $S$, then the functions

$$
\kappa_{g}(s)=\left\langle\mathbf{t}^{\prime}(s), \mathbf{U}(s)\right\rangle_{1}, \quad \bar{\kappa}_{n}(s)=\left\langle\mathbf{t}^{\prime}(s), \zeta(s)\right\rangle_{1}, \quad \bar{\tau}_{g}(s)=\left\langle\zeta^{\prime}(s), \mathbf{U}(s)\right\rangle_{1},
$$

are called the geodesic curvature, pseudo normal curvature and pseudo geodesic torsion of the curve $\alpha(s)$, respectively.

Theorem 2. Let $S$ be a lightlike surface in $\mathbb{R}_{1}^{3}$ and $\alpha(s)$ be a curve on $S$. Then derivative formulas of the natural frame $\{\mathbf{t}, \mathbf{N}, \mathbf{U}\}$ are given by

$$
\left[\begin{array}{c}
\mathbf{t}^{\prime} \\
\mathbf{N}^{\prime} \\
\mathbf{U}^{\prime}
\end{array}\right]=\left[\begin{array}{ccc}
0 & \kappa_{g} & \bar{\kappa}_{n} \\
-\bar{\kappa}_{n} & \bar{\tau}_{g} & 0 \\
-\kappa_{g} & 0 & -\bar{\tau}_{g}
\end{array}\right]\left[\begin{array}{c}
\mathbf{t} \\
\mathbf{N} \\
\mathbf{U}
\end{array}\right]
$$

4.3.3. Darboux Frame of Null Curves on Timelike Surfaces. Let $S$ be a timelike surface in $\mathbb{R}_{1}^{3}$, $\alpha: I \rightarrow S \subset \mathbb{R}_{1}^{3}$ be a distinguished null curve, $\mathbf{N}$ be the normal vector field of the surface $S$ and $\mathbf{t}$ is the tangent vector field of the curve $\alpha(s)$, then there exists only one Darboux frame (null frame) $\{\mathbf{N}, \mathbf{t}, \mathbf{U}\}$, such that $\langle\mathbf{t}, \mathbf{t}\rangle_{1}=\langle\mathbf{U}, \mathbf{U}\rangle_{1}=\langle\mathbf{t}, \mathbf{N}\rangle_{1}=\langle\mathbf{N}, \mathbf{U}\rangle_{1}=0,\langle\mathbf{N}, \mathbf{N}\rangle_{1}=\langle\mathbf{t}, \mathbf{U}\rangle_{1}=1, \mathbf{t} \times{ }_{1} \mathbf{U}=\mathbf{N}, \mathbf{N} \times{ }_{1} \mathbf{t}=\mathbf{t}$, $\mathbf{U} \times{ }_{1} \mathbf{N}=\mathbf{U}$, this frame is a pseudo-orthonormal basis in $\mathbb{R}_{1}^{3}$. Also, this frame satisfies (3.1) which can be constructed as in section 3.1 .3 , so it is given by

$$
\mathbf{t}=\left(x^{\prime}, y^{\prime}, z^{\prime}\right), \quad \mathbf{N}=\left(N^{1}, N^{2}, N^{3}\right), \quad \mathbf{U}=-\frac{v}{2 x^{\prime 2}}\left[\left(2 v x^{\prime}, 2 N^{1} z^{\prime},-2 N^{1} y^{\prime}\right)+v\left(\left(N^{1}\right)^{2}-1\right) \mathbf{t}\right],
$$

where

$$
v=\frac{N^{2} z^{\prime}-N^{3} y^{\prime}}{x^{\prime}} .
$$

Theorem 3. Let $S$ be a timelike surface in $\mathbb{R}_{1}^{3}$ and $\alpha(s)$ be a curve lies on the surface $S$. Then derivative formulas of the natural frame $\{\mathbf{N}, \mathbf{t}, \mathbf{U}\}$ are given by

$$
\left[\begin{array}{c}
\mathbf{N}^{\prime} \\
\mathbf{t}^{\prime} \\
\mathbf{U}^{\prime}
\end{array}\right]=\left[\begin{array}{ccc}
0 & \tau_{g} & -\kappa_{n} \\
\kappa_{n} & \kappa_{g} & 0 \\
-\tau_{g} & 0 & -\kappa_{g}
\end{array}\right]\left[\begin{array}{c}
\mathbf{N} \\
\mathbf{t} \\
\mathbf{U}
\end{array}\right]
$$

where

$$
\kappa_{g}(s)=\left\langle\mathbf{t}^{\prime}(s), \mathbf{U}(s)\right\rangle_{1}, \kappa_{n}(s)=\left\langle\mathbf{t}^{\prime}(s), \mathbf{N}(s)\right\rangle_{1}, \tau_{g}(s)=\left\langle\mathbf{N}^{\prime}(s), \mathbf{U}(s)\right\rangle_{1},
$$

which are called the geodesic curvature, normal curvature and geodesic torsion of the curve $\alpha(s)$, respectively.

4.3.4. Darboux Frame of Null Curves on Lightlike Surfaces. If $\{\mathbf{t}, \mathbf{n}, \mathbf{b}\}$ be the null Frenet frame of a null curve $\alpha(\mathbf{s})$ which lies on a lightlike surface $S$ in $\mathbb{R}_{1}^{3}$. In this case the tangent vector field $\mathbf{t}$ of the curve $\alpha(\mathbf{s})$ and the normal vector field $\zeta$ of the surface $S$ are linearly dependent $(\mathbf{N}=\omega \mathbf{t} ; \omega \in \mathbb{R}-\{0\})$ and the Darboux frame is not defined $\left(\{\mathbf{t}, \mathbf{N}, \mathbf{U}\}\right.$ where $\left.\langle\mathbf{t}, \mathbf{N}\rangle_{1}=0\right)$.

5. Transversal Intersection Curves of Two Surfaces in $\mathbb{R}_{1}^{3}$. The intersection curve of two spacelike surfaces is a spacelike, the intersection curve of two timelike surfaces may be spacelike or timelike, the intersection curve of two lightlike surfaces may be spacelike or null, the intersection curve of spacelike and timelike surfaces is spacelike, the intersection curve of spacelike and lightlike surfaces is spacelike, and the intersection curve of timelike and lightlike surfaces spacelike or null. We obtain the transversal intersection case, when the normal vectors of the surfaces are linearly independent.

For what follows we suppose $\alpha(s)$ be a transversal intersection curve of two arbitrary surfaces $\mathbf{S}^{A}$ and $\mathbf{S}^{B}$ and assume that the curve $\beta(u)=\alpha(s(u))$ is a regular curve parametrized by a parameter $u$ with the same trace as the curve $\alpha(s)$. Let $\zeta^{A}$ and $\zeta^{B}$ denote the normal vector fields of the surfaces $S^{A}$ and $S^{B}$, respectively. Also, let $\mathbf{N}^{A}$ and $\mathbf{N}^{B}$ denote the unit normal vector fields of the surfaces $S^{A}$ and $S^{B}$, respectively. Assume that $\left\langle\zeta^{A}, \zeta^{B}\right\rangle_{1}=\lambda,\left\langle\zeta^{A}, \zeta^{A}\right\rangle_{1}=\varepsilon^{A},\left\langle\zeta^{B}, \zeta^{B}\right\rangle_{1}=\varepsilon^{B},\left\langle\mathbf{N}^{A}, \mathbf{N}^{B}\right\rangle_{1}=\bar{\lambda}$, $\left\langle\mathbf{N}^{A}, \mathbf{N}^{A}\right\rangle_{1}=\bar{\varepsilon}^{A},\left\langle\mathbf{N}^{B}, \mathbf{N}^{B}\right\rangle_{1}=\bar{\varepsilon}^{B}$.

Since $\zeta^{A} \times{ }_{1} \zeta^{B}$ is orthogonal to $\zeta^{A}$ and $\zeta^{B}$, so by using proposition 2, we obtain

$$
\left\langle\zeta^{A} \times_{1} \zeta^{B}, \zeta^{A} \times_{1} \zeta^{B}\right\rangle_{1}=\left|\begin{array}{cc}
\lambda & \varepsilon^{B} \\
\varepsilon^{A} & \lambda
\end{array}\right|=\lambda^{2}-\varepsilon^{A} \varepsilon^{B} .
$$

The surfaces are intersect transversely if $\lambda^{2}-\varepsilon^{A} \varepsilon^{B} \neq 0$. In particular, if $\lambda=0, \varepsilon^{A}=0$ and $\varepsilon^{B}>0$, in this case the surfaces are intersect transversely at a null curve. 


\subsection{The Tangent Vector Field.}

- If $\lambda^{2}-\varepsilon^{A} \varepsilon^{B} \neq 0$, then The tangent vector field of the curve $\beta(u)$ is given by $\beta^{\prime}(u)=\zeta^{A} \times{ }_{1} \zeta^{B}$, so that the unit tangent vector field $\mathbf{t}$ of the transversal intersection curve is given by

$$
\alpha^{\prime}(s)=\frac{\zeta^{A} \times{ }_{1} \zeta^{B}}{\left\|\zeta^{A} \times{ }_{1} \zeta^{B}\right\|_{1}} .
$$

- If $\lambda=0, \varepsilon^{A}=0, \varepsilon^{B}=1$, then the tangent vector field of the transversal intersection curve is null. Moreover, $\alpha^{\prime}(s)$ and $\zeta^{A}$ are linearly dependent.

From the foregoing results, we have the following preposition:

Proposition 4. For the transversal intersection curve of two surfaces, we get.

i) The transversal intersection curve of two spacelike or two timelike surfaces is spacelike or timelike curve if, $\bar{\lambda} \in \mathbb{R}-[-1,1]$ or $\bar{\lambda} \in]-1,1[$, respectively.

ii) The transversal intersection curve of two lightlike surfaces is spacelike curve.

iii) The transversal intersection curve of a spacelike and a timelike surfaces is spacelike curve.

iv) The transversal intersection curve of a lightlike surface and a spacelike surface is spacelike curve.

v) The transversal intersection curve of a lightlike surface and a timelike surface is spacelike or lightlike curve if, $\lambda \neq 0$ or $\lambda=0$, respectively.

\subsection{The Second Order Derivative Vector Field.}

- If $\lambda^{2}-\varepsilon^{A} \varepsilon^{B} \neq 0$, the vector field $\alpha^{\prime \prime}(s)$ must lies in the normal plane spanned by $\zeta^{A}$ and $\zeta^{B}$, thus we can write,

$$
\alpha^{\prime \prime}(s)=\beta_{1} \zeta^{A}+\beta_{2} \zeta^{B},
$$

where $\beta_{1}$ and $\beta_{2}$ are unknown coefficients that we need to determine. Projecting $\alpha^{\prime \prime}(s)$ onto the normal vector fields of both surfaces and solving them for the unknown coefficients and substitution into (5.2), we get

$$
\alpha^{\prime \prime}(s)=\frac{\varepsilon^{B} \bar{\kappa}_{n}^{A}-\lambda \bar{\kappa}_{n}^{B}}{\varepsilon^{A} \varepsilon^{B}-\lambda^{2}} \zeta^{A}+\frac{\varepsilon^{A} \bar{\kappa}_{n}^{B}-\lambda \bar{\kappa}_{n}^{A}}{\varepsilon^{A} \varepsilon^{B}-\lambda^{2}} \zeta^{B},
$$

where $\bar{\kappa}_{n}^{A}=\left\langle\alpha^{\prime \prime}(s), \zeta^{A}\right\rangle_{1}$ and $\bar{\kappa}_{n}^{B}=\left\langle\alpha^{\prime \prime}(s), \zeta^{B}\right\rangle_{1}$ and obtained by (4.3) or (4.14).

- If $\lambda=0, \varepsilon^{A}=0, \bar{\varepsilon}^{B}=1$, then $\alpha(s)$ is a null curve. If $\alpha^{\prime \prime}(s) \neq 0$, then $\alpha^{\prime}(s)$ and $\zeta^{A}$ have the same direction, $\left\langle\alpha^{\prime}(s), \zeta^{A}\right\rangle_{1}=0,\left\langle\alpha^{\prime}(s), \alpha^{\prime \prime}(s)\right\rangle_{1}=0$ and $\left\langle\alpha^{\prime \prime}(s), \alpha^{\prime \prime}(s)\right\rangle_{1}=1$. In this case we can assume the null frame $\left\{\mathbf{N}^{B}, \zeta^{A}, \mathbf{U}\right\}$, then the vector $\alpha^{\prime \prime}(s)$ can write

$$
\alpha^{\prime \prime}(s)=\mathbf{N}^{B} .
$$

The third vector of the null frame $\mathbf{U}$ can be constructed as in section 3.1.2, so it is given by

$$
\mathbf{U}=-\frac{\varrho}{2\left(\zeta^{1 A}\right)^{2}}\left(\left(2 \varrho \zeta^{1 A}, 2 N^{1 B} \zeta^{3 A},-2 N^{1 B} \zeta^{2 A}\right)+\varrho\left(\left(N^{1 B}\right)^{2}-1\right) \zeta^{A}\right),
$$

where

$$
\zeta^{A}=\left(\zeta^{1 A}, \zeta^{2 A}, \zeta^{3 A}\right), \quad \mathbf{N}^{B}=\left(N^{1 B}, N^{2 B}, N^{3 B}\right), \quad \varrho=\frac{N^{2 B} \zeta^{3 A}-N^{3 B} \zeta^{2 A}}{\zeta^{1 A}} .
$$

\subsection{The Third Order Derivative Vector Field.}

- If $\lambda^{2}-\varepsilon^{A} \varepsilon^{B} \neq 0$ and $\alpha^{\prime \prime}(s)$ is not lightlike, differentiating the vector field $\alpha^{\prime \prime}(s)$ and using (3.5), we get

$$
\alpha^{\prime \prime \prime}(s)=-\epsilon_{1} \epsilon_{2}^{2} k^{2} \mathbf{t}+\left(\left(\epsilon_{2}^{2}-1\right) k \tau+k^{\prime}\right) \mathbf{n}-\epsilon_{3} k \tau \mathbf{b},
$$

Since the vectors $\zeta^{A}$ and $\zeta^{B}$ lie on the normal plane of the unit tangent vector $\mathbf{t}$, then we can write

$$
\alpha^{\prime \prime \prime}(s)=-\epsilon_{1} \epsilon_{2}^{2} \kappa^{2} \mathbf{t}+\beta_{5} \zeta^{A}+\beta_{6} \zeta^{B},
$$

where $\beta_{5}$ and $\beta_{6}$ are unknown coefficients that we need to determine. Projecting the vector field $\alpha^{\prime \prime \prime}(s)$ onto the normal vector fields of both surfaces, solving for unknown coefficients and substituting into (5.4) we obtain,

$$
\alpha^{\prime \prime \prime}(s)=-\epsilon_{1} \epsilon_{3}^{2} \kappa^{2} \mathbf{t}+\frac{\varepsilon^{B} \bar{p}_{n}^{A}-\lambda \bar{p}_{n}^{B}}{\varepsilon^{A} \varepsilon^{B}-\lambda^{2}} \zeta^{A}+\frac{\varepsilon^{A} \bar{p}_{n}^{B}-\lambda \bar{p}_{n}^{A}}{\varepsilon^{A} \varepsilon^{B}-\lambda^{2}} \zeta^{B},
$$

where $\bar{p}_{n}^{A}=\left\langle\alpha^{\prime \prime \prime}(s), \zeta^{A}\right\rangle_{1}, \bar{p}_{n}^{B}=\left\langle\alpha^{\prime \prime \prime}(s), \zeta^{B}\right\rangle_{1}$ and obtained by (4.4) or (4.15). 
- If $\lambda^{2}-\varepsilon^{A} \varepsilon^{B} \neq 0$ and $\alpha^{\prime \prime}(s)$ is lightlike, differentiating the vector field $\alpha^{\prime \prime}(s)$ and using (3.10), we get

$$
\alpha^{\prime \prime \prime}(s)=\beta_{7} \zeta^{A}+\beta_{8} \zeta^{B}
$$

where $\beta_{7}$ and $\beta_{8}$ are unknown coefficients that we need to determine. Projecting the vector field $\alpha^{\prime \prime \prime}(s)$ onto the normal vector fields of both surfaces, solving for unknown coefficients and substituting into (5.6) we obtain,

$$
\alpha^{\prime \prime \prime}(s)=\frac{\varepsilon^{B} \bar{p}_{n}^{A}-\lambda \bar{p}_{n}^{B}}{\varepsilon^{A} \varepsilon^{B}-\lambda^{2}} \zeta^{A}+\frac{\varepsilon^{A} \bar{p}_{n}^{B}-\lambda \bar{p}_{n}^{A}}{\varepsilon^{A} \varepsilon^{B}-\lambda^{2}} \zeta^{B},
$$

where $\bar{p}_{n}^{A}=\left\langle\alpha^{\prime \prime \prime}(s), \zeta^{A}\right\rangle_{1}, \bar{p}_{n}^{B}=\left\langle\alpha^{\prime \prime \prime}(s), \zeta^{B}\right\rangle_{1}$ and obtained by (4.4) or (4.15).

- If $\lambda=0, \varepsilon^{A}=0, \bar{\varepsilon}^{B}=1$, then the vector $\alpha^{\prime \prime \prime}(s)$ can be written as

$$
\alpha^{\prime \prime \prime}(s)=\beta_{9} \zeta^{A}+\beta_{10} \mathbf{U}
$$

where $\beta_{9}$ and $\beta_{10}$ are unknown coefficients that we need to determine. Projecting the vector field $\alpha^{\prime \prime \prime}(s)$ onto the vectors $\zeta^{A}, \mathbf{U}$ and $\mathbf{N}^{B}$, solving for unknown coefficients and substituting in (5.8), we obtain

$$
\alpha^{\prime \prime \prime}(s)=\bar{p}_{n}^{U} \zeta^{A}+\bar{p}_{n}^{A} \mathbf{U}
$$

where $\bar{p}_{n}^{A}=\left\langle\alpha^{\prime \prime \prime}(s), \zeta^{A}\right\rangle_{1}$ is obtained by (4.4) or (4.15) and $\bar{p}_{n}^{U}=\left\langle\alpha^{\prime \prime \prime}(u), \mathbf{U}\right\rangle_{1}$.

6. Characterizations of Transversal Intersection curves of two Surfaces in $\mathbb{R}_{1}^{3}$. The characterizations of the transversal intersection curves of two spacelike, two timelike and spacelike-timelike surfaces can be found in [6], [22] and [16], respectively. In this section, we introduce the characterization of intersection curves of two lightlike, lightlike-timelike and lightlike-spacelike surfaces.

Theorem 4. Let $\alpha=\alpha(s)$ be the transversal intersection curve of two lightlike surfaces $\mathbf{S}^{A}$ and $\mathbf{S}^{B}$. The geodesic curvatures of curve are given by

$$
k_{g}^{A}=\frac{\bar{\kappa}_{n}^{B}}{\lambda}, \quad k_{g}^{B}=\frac{\bar{\kappa}_{n}^{A}}{\lambda} .
$$

If $\alpha^{\prime \prime}(s)$ is not lightlike, then the curvature and the torsion are given by

$$
k^{2}=\left|\frac{2 \bar{\kappa}_{n}^{A} \bar{\kappa}_{n}^{B}}{\lambda^{2}}\right|, \quad \tau=-\frac{\epsilon_{2}\left(\bar{p}_{n}^{B}\left\langle\zeta^{A}, \mathbf{b}\right\rangle_{1}+\bar{p}_{n}^{B}\left\langle\zeta^{B}, \mathbf{b}\right\rangle_{1}\right)}{k \lambda} .
$$

If $\alpha^{\prime \prime}(s)$ is lightlike, then the torsion is given by

$$
\tau=-\frac{\bar{p}_{n}^{B}\left\langle\zeta^{A}, \mathbf{b}\right\rangle_{1}+\bar{p}_{n}^{B}\left\langle\zeta^{B}, \mathbf{b}\right\rangle_{1}}{\lambda}
$$

Proof: In this case the intersection curve is spacelike. To write the geodesic curvatures as a function of the normal curvatures, we need to write the vector $\mathbf{U}^{A}, \mathbf{U}^{B}$ as a linear combination of $\zeta^{A}$ and $\zeta^{B}$ and applied in the equation (4.19). Thus,

$$
\mathbf{U}^{A}=x_{0} \zeta^{A}+y_{0} \zeta^{B}, \quad \mathbf{U}^{B}=x_{1} \zeta^{A}+y_{1} \zeta^{B},
$$

then we have

$$
\begin{aligned}
& \left\{\begin{array} { c } 
{ 1 = \langle \mathbf { U } ^ { A } , \zeta ^ { A } \rangle _ { 1 } = x _ { 0 } \langle \zeta ^ { A } , \zeta ^ { A } \rangle _ { 1 } + y _ { 0 } \langle \zeta ^ { B } , \zeta ^ { A } \rangle _ { 1 } } \\
{ 0 = \langle \mathbf { U } ^ { A } , \mathbf { U } ^ { A } \rangle _ { 1 } = ( x _ { 0 } ) ^ { 2 } \langle \zeta ^ { A } , \zeta ^ { A } \rangle _ { 1 } + 2 x _ { 0 } y _ { 0 } \langle \zeta ^ { B } , \zeta ^ { A } \rangle _ { 1 } + ( y _ { 0 } ) ^ { 2 } \langle \zeta ^ { B } , \zeta ^ { B } \rangle _ { 1 } }
\end{array} \quad \Longrightarrow \left\{\begin{array}{c}
y_{0}=\frac{1}{\lambda} \\
x_{0}=0
\end{array},\right.\right. \\
& \left\{\begin{array} { c } 
{ 1 = \langle \mathbf { U } ^ { B } , \zeta ^ { B } \rangle _ { 1 } = x _ { 1 } \langle \zeta ^ { A } , \zeta ^ { B } \rangle _ { 1 } + y _ { 1 } \langle \zeta ^ { B } , \zeta ^ { B } \rangle _ { 1 } } \\
{ 0 = \langle \mathbf { U } ^ { B } , \mathbf { U } ^ { B } \rangle _ { 1 } = ( x _ { 1 } ) ^ { 2 } \langle \zeta ^ { A } , \zeta ^ { A } \rangle _ { 1 } + 2 x _ { 1 } y _ { 1 } \langle \zeta ^ { B } , \zeta ^ { A } \rangle _ { 1 } + ( y _ { 1 } ) ^ { 2 } \langle \zeta ^ { B } , \zeta ^ { B } \rangle _ { 1 } }
\end{array} \quad \Longrightarrow \left\{\begin{array}{c}
x_{1}=\frac{1}{\lambda} \\
y_{1}=0
\end{array} .\right.\right.
\end{aligned}
$$


If $\alpha^{\prime \prime}(s)$ is not lightlike the curvature $\kappa$ is obtained using the equation (5.3), $\kappa^{2}=\left\langle\alpha^{\prime \prime}, \alpha^{\prime \prime}\right\rangle_{1}$ and the torsion is obtained using the equations (3.4) and (5.5). If $\alpha^{\prime \prime}(s)$ is lightlike the curvature is not defined and the torsion is obtained using the equations (3.11) and (5.7). $\square$

Theorem 5. Let $\alpha=\alpha(s)$ be the transversal intersection curve of a lightlike surface $S^{A}$ and a spacelike surface $S^{B}$. The geodesic curvatures of intersection curve are given by

$$
\kappa_{g}^{A}=\frac{\left\|\zeta^{B}\right\|^{2} \bar{\kappa}_{n}^{A}+2 \lambda\left\|\zeta^{B}\right\| \kappa_{n}^{B}}{2 \lambda^{2}}, \quad \kappa_{g}^{B}=\frac{\left\|\zeta^{B}\right\| \bar{\kappa}_{n}^{A}+\lambda \kappa_{n}^{B}}{|\lambda|} .
$$

If $\alpha^{\prime \prime}(s)$ is not lightlike, then the curvature and the torsion are given by

$$
k^{2}=\left|\frac{\left(\bar{\kappa}_{n}^{A}\right)^{2}-2 \lambda \bar{\kappa}_{n}^{A} \kappa_{n}^{B}}{\lambda^{2}}\right|, \quad \tau=-\frac{\epsilon_{2}}{k}\left(\frac{-\varepsilon^{B} \bar{p}_{n}^{A}+\lambda \bar{p}_{n}^{B}}{\lambda^{2}}\left\langle\zeta^{A}, \mathbf{b}\right\rangle_{1}+\frac{\bar{p}_{n}^{B}}{\lambda}\left\langle\zeta^{B}, \mathbf{b}\right\rangle_{1}\right) .
$$

If $\alpha^{\prime \prime}(s)$ is lightlike, then the torsion is given by

$$
\tau=-\frac{-\varepsilon^{B} \bar{p}_{n}^{A}+\lambda \bar{p}_{n}^{B}}{\lambda^{2}}\left\langle\zeta^{A}, \mathbf{b}\right\rangle_{1}-\frac{\bar{p}_{n}^{B}}{\lambda}\left\langle\zeta^{B}, \mathbf{b}\right\rangle_{1} .
$$

Proof: In this case the intersection curve is spacelike. To write the geodesic curvatures as a function of the normal curvatures, we need to write the vector $\mathbf{U}^{A}, \mathbf{U}^{B}$ as a linear combination of $\zeta^{A}$ and $\zeta^{B}$ and applied in the equations (4.17) and (4.19). Thus,

$$
\mathbf{U}^{A}=x_{0} \zeta^{A}+y_{0} \zeta^{B}
$$

then we have

$$
\begin{aligned}
& \left\{\begin{array}{c}
1=\left\langle\mathbf{U}^{A}, \zeta^{A}\right\rangle_{1}=x_{0}\left\langle\zeta^{A}, \zeta^{A}\right\rangle_{1}+y_{0}\left\langle\zeta^{B}, \zeta^{A}\right\rangle_{1} \\
0=\left\langle\mathbf{U}^{A}, \mathbf{U}^{A}\right\rangle_{1}=\left(x_{0}\right)^{2}\left\langle\zeta^{A}, \zeta^{A}\right\rangle_{1}+2 x_{0} y_{0}\left\langle\zeta^{B}, \zeta^{A}\right\rangle_{1}+\left(y_{0}\right)^{2}\left\langle\zeta^{B}, \zeta^{B}\right\rangle_{1}
\end{array}\right. \\
& \Longrightarrow\left\{\begin{array} { c } 
{ 1 = \langle \mathbf { U } ^ { A } , \zeta ^ { A } \rangle _ { 1 } = y _ { 0 } \lambda } \\
{ 0 = \langle \mathbf { U } ^ { A } , \mathbf { U } ^ { A } \rangle _ { 1 } = 2 x _ { 0 } y _ { 0 } \lambda + ( y _ { 0 } ) ^ { 2 } \varepsilon ^ { B } \| \zeta ^ { B } \| ^ { 2 } }
\end{array} \Longrightarrow \left\{\begin{array}{c}
y_{0}=\frac{1}{\lambda} \\
x_{0}=-\frac{\varepsilon^{B}\left\|\zeta^{B}\right\|^{2}}{2 \lambda^{2}} .
\end{array}\right.\right. \\
& \Longrightarrow \mathbf{U}^{A}=-\frac{\varepsilon^{B}\left\|\zeta^{B}\right\|^{2}}{2 \lambda^{2}} \zeta^{A}+\frac{\left\|\zeta^{B}\right\| \mathbf{N}^{B}}{\lambda} .
\end{aligned}
$$

Also, we have

$$
\begin{aligned}
\mathbf{U}^{B} & =\mathbf{N}^{B} \times_{1} \mathbf{t} \\
& =\frac{\mathbf{N}^{B} \times_{1}\left(\zeta^{A} \times{ }_{1} \zeta^{B}\right)}{\left\|\zeta^{A} \times_{1} \zeta^{B}\right\|} \\
& =\frac{-\left\langle\zeta^{B}, \mathbf{N}^{B}\right\rangle_{1} \zeta^{A}+\left\langle\zeta^{A}, \mathbf{N}^{B}\right\rangle_{1} \zeta^{B}}{\left\|\zeta^{A} \times{ }_{1} \zeta^{B}\right\|} \\
& =\frac{-\left\|\zeta^{B}\right\|\left\langle\mathbf{N}^{B}, \mathbf{N}^{B}\right\rangle_{1} \zeta^{A}+\left\langle\zeta^{A}, \frac{\zeta^{B}}{\left\|\zeta^{B}\right\|}\right\rangle_{1} \zeta^{B}}{\left\|\zeta^{A} \times \zeta_{1}^{B}\right\|} \\
& =\frac{1}{\left\|\zeta^{A} \times \zeta_{1} \zeta^{B}\right\|}\left(-\left\|\zeta^{B}\right\|\left\langle\mathbf{N}^{B}, \mathbf{N}^{B}\right\rangle_{1} \zeta^{A}+\frac{1}{\left\|\zeta^{B}\right\|}\left\langle\zeta^{A}, \zeta^{B}\right\rangle_{1} \zeta^{B}\right) \\
& =\frac{1}{\left\|\zeta^{A} \times{ }_{1} \zeta^{B}\right\|}\left(-\left\|\zeta^{B}\right\| \varepsilon^{B} \zeta^{A}+\lambda \frac{\zeta^{B}}{\left\|\zeta^{B}\right\|}\right) .
\end{aligned}
$$

If $\alpha^{\prime \prime}(s)$ is not lightlike the curvature $\kappa$ is obtained using the equation (5.3), $\kappa^{2}=\left\langle\alpha^{\prime \prime}, \alpha^{\prime \prime}\right\rangle_{1}$ and the torsion is obtained using the equations (3.4) and (5.5). If $\alpha^{\prime \prime}(s)$ is lightlike the curvature is not defined and the torsion is obtained using the equations (3.11) and (5.7).

Theorem 6. Let $\alpha=\alpha(s)$ be the transversal intersection curve of a lightlike surface $S^{A}$ and a timelike surface $S^{B}$. If $\alpha^{\prime}(s)$ is lightlike, then the torsion is given by

$$
\tau=-\bar{p}_{n}^{U}\left\langle\zeta^{A}, \mathbf{b}\right\rangle_{1}-\bar{p}_{n}^{A}\langle\mathbf{U}, \mathbf{b}\rangle_{1} .
$$


If $\alpha^{\prime}(s)$ is spacelike the geodesic curvatures of intersection curve are given by

$$
\kappa_{g}^{A}=\frac{\left\|\zeta^{B}\right\|^{2} \bar{\kappa}_{n}^{A}+2 \lambda\left\|\zeta^{B}\right\| \kappa_{n}^{B}}{2 \lambda^{2}}, \quad \kappa_{g}^{B}=\frac{-\left\|\zeta^{B}\right\| \bar{\kappa}_{n}^{A}+\lambda \kappa_{n}^{B}}{|\lambda|} .
$$

If $\alpha^{\prime}(s)$ is spacelike and $\alpha^{\prime \prime}(s)$ is not lightlike, then the curvature and the torsion are given by

$$
k^{2}=\left|\frac{\left(\bar{\kappa}_{n}^{A}\right)^{2}+2 \lambda \bar{\kappa}_{n}^{A} \kappa_{n}^{B}}{\lambda^{2}}\right|, \quad \tau=-\frac{\epsilon_{2}}{k}\left(\frac{-\varepsilon^{B} \bar{p}_{n}^{A}+\lambda \bar{p}_{n}^{B}}{\lambda^{2}}\left\langle\zeta^{A}, \mathbf{b}\right\rangle_{1}+\frac{\bar{p}_{n}^{B}}{\lambda}\left\langle\zeta^{B}, \mathbf{b}\right\rangle_{1}\right) .
$$

If $\alpha^{\prime}(s)$ is spacelike and $\alpha^{\prime \prime}(s)$ is lightlike, then the torsion of $\alpha(s)$ is given by

$$
\tau=-\frac{-\varepsilon^{B} \bar{p}_{n}^{A}+\lambda \bar{p}_{n}^{B}}{\lambda^{2}}\left\langle\zeta^{A}, \mathbf{b}\right\rangle_{1}-\frac{\bar{p}_{n}^{B}}{\lambda}\left\langle\zeta^{B}, \mathbf{b}\right\rangle_{1}
$$

Proof: If $\alpha^{\prime}(s)$ is lightlike by section 4.3.4 Darboux frame is not defined to $S^{A}$ and torsion is obtained using the equations (3.13) and (5.9).

Suppose the intersection curve is spacelike. To write the geodesic curvatures as a function of the normal curvatures, we need to write the vector $\mathbf{U}^{A}, \mathbf{U}^{B}$ as a linear combination of $\zeta^{A}$ and $\zeta^{B}$ and applied in the equations (4.17) and (4.19). Thus,

$$
\mathbf{U}^{A}=x_{0} \zeta^{A}+y_{0} \zeta^{B}
$$

then we have

$$
\begin{gathered}
\left\{\begin{array}{c}
1=\left\langle\mathbf{U}^{A}, \zeta^{A}\right\rangle_{1}=x_{0}\left\langle\zeta^{A}, \zeta^{A}\right\rangle_{1}+y_{0}\left\langle\zeta^{B}, \zeta^{A}\right\rangle_{1} \\
0=\left\langle\mathbf{U}^{A}, \mathbf{U}^{A}\right\rangle_{1}=\left(x_{0}\right)^{2}\left\langle\zeta^{A}, \zeta^{A}\right\rangle_{1}+2 x_{0} y_{0}\left\langle\zeta^{B}, \zeta^{A}\right\rangle_{1}+\left(y_{0}\right)^{2}\left\langle\zeta^{B}, \zeta^{B}\right\rangle_{1}
\end{array}\right. \\
\Longrightarrow\left\{\begin{array}{c}
y_{0}=\frac{1}{\lambda} \\
x_{0}=-\frac{\varepsilon^{B}\left\|\zeta^{B}\right\|^{2}}{2 \lambda^{2}}
\end{array} \Longrightarrow \mathbf{U}^{A}=-\frac{\varepsilon^{B}\left\|\zeta^{B}\right\|^{2}}{2 \lambda^{2}} \zeta^{A}+\frac{1}{\lambda} \zeta^{B} .\right.
\end{gathered}
$$

Also we have

$$
\begin{aligned}
\mathbf{U}^{B} & =\mathbf{N}^{B} \times_{1} \mathbf{t} \\
& =\frac{\mathbf{N}^{B} \times_{1}\left(\zeta^{A} \times{ }_{1} \zeta^{B}\right)}{\left\|\zeta^{A} \times_{1} \zeta^{B}\right\|} \\
& =\frac{-\left\langle\zeta^{B}, \mathbf{N}^{B}\right\rangle_{1} \zeta^{A}+\left\langle\zeta^{A}, \mathbf{N}^{B}\right\rangle_{1} \zeta^{B}}{\left\|\zeta^{A} \times{ }_{1} \zeta^{B}\right\|} \\
& =\frac{-\left\|\zeta^{B}\right\|\left\langle\mathbf{N}^{B}, \mathbf{N}^{B}\right\rangle_{1} \zeta^{A}+\left\langle\zeta^{A}, \frac{\zeta^{B}}{\left\|\zeta^{B}\right\|}\right\rangle_{1} \zeta^{B}}{\left\|\zeta^{A} \times_{1} \zeta^{B}\right\|} \\
& =\frac{1}{\left\|\zeta^{A} \times_{1} \zeta^{B}\right\|}\left(-\left\|\zeta^{B}\right\|\left\langle\mathbf{N}^{B}, \mathbf{N}^{B}\right\rangle_{1} \zeta^{A}+\frac{1}{\left\|\zeta^{B}\right\|}\left\langle\zeta^{A}, \zeta^{B}\right\rangle_{1} \zeta^{B}\right) \\
& =\frac{1}{\left\|\zeta^{A} \times_{1} \zeta^{B}\right\|}\left(-\left\|\zeta^{B}\right\| \varepsilon^{B} \zeta^{A}+\lambda \frac{\zeta^{B}}{\left\|\zeta^{B}\right\|}\right)
\end{aligned}
$$

If $\alpha^{\prime \prime}(s)$ is not lightlike the curvature $\kappa$ is obtained using the equation (5.3), $\kappa^{2}=\left\langle\alpha^{\prime \prime}, \alpha^{\prime \prime}\right\rangle_{1}$ and the torsion is obtained using the equations (3.4) and (5.5). If $\alpha^{\prime \prime}(s)$ is lightlike the curvature is not defined and the torsion is obtained using the equations (3.11) and (5.7).

7. Examples. In this section we presentation some examples of transversal intersection curves of two surfaces and we compute its Frenet frame, Darboux frame, curvature (when exists), torsion, normal curvature and geodesic curvature in a point fixed.

Example 1. Consider the two implicit lightlike surfaces $S^{A}$ and $S^{B}$ as in the following

$$
S^{A}: \quad f\left(x_{1}, x_{2}, x_{3}\right)=\sqrt{2} x_{1}-x_{2}-x_{3}-1=0, \quad S^{B}: h\left(x_{1}, x_{2}, x_{3}\right)=x_{1}^{2}-x_{2}^{2}-x_{3}^{2}=0 .
$$




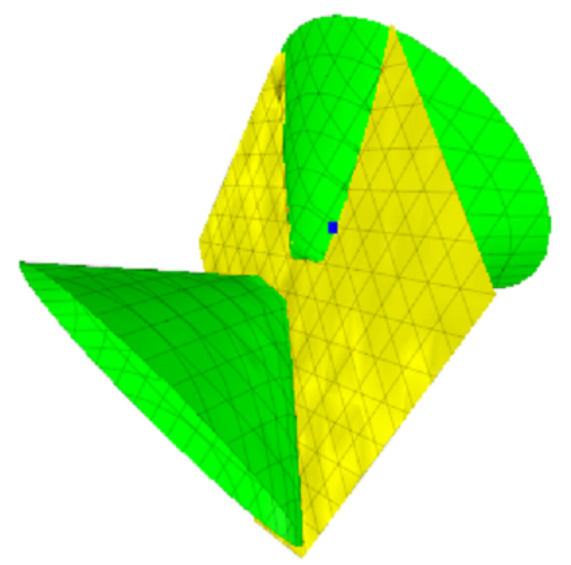

FIGURE 7.1. Transversal intersection of two implicit lightlike surfaces

Using (7.1), we obtain $\zeta^{A}=(-\sqrt{2},-1,-1), \zeta^{B}=\left(-2 x_{1},-2 x_{2},-2 x_{3}\right)$, therefore $\varepsilon^{A}=\varepsilon^{B}=0$, for any point on the surfaces, thus $S^{A}$ and $S^{B}$ are lightlike surfaces. Considering the intersection point $p_{0}=\left(\sqrt{2}, \frac{1-\sqrt{3}}{2}, \frac{1+\sqrt{3}}{2}\right)$, (see Figure 1 ), in this point we have, $\lambda=-2 \neq 0$ (transversal intersection). Using (4.13), (4.15),(5.1), (5.3), (5.7) and (7.1) at the intersection point $p_{0}$, we obtain

$$
\mathbf{t}=\boldsymbol{\alpha}^{\prime}=\left(-\sqrt{3}, \frac{1}{2} \sqrt{2}-\frac{1}{2} \sqrt{6},-\frac{1}{2} \sqrt{2}-\frac{1}{2} \sqrt{6}\right), \quad \boldsymbol{\alpha}^{\prime \prime}=(\sqrt{2}, 1,1), \quad \boldsymbol{\alpha}^{\prime \prime \prime}=(0,0,0) .
$$

Thus, the intersection curve at this point is pseudo null. Using, (3.9), (3.11), (3.12) and (7.2), we obtain

$$
\mathbf{n}=(\sqrt{2}, 1,1) \quad \mathbf{b}=-\frac{1}{2}(2 \sqrt{2}, 1-\sqrt{3}, 1+\sqrt{3}), \quad \tau=0 .
$$

Using (4.18) and (7.2) we obtain

$$
\mathbf{U}^{A}=\frac{1}{2}(2 \sqrt{2}, 1-\sqrt{3}, 1+\sqrt{3}), \quad \mathbf{U}^{B}=\frac{1}{2}(\sqrt{2}, 1,1)
$$

Using (4.19), (6.1) and (6.2), then the geodesic curvature, pseudo normal curvature and pseudo geodesic torsion are given by

$$
\bar{\kappa}_{n}^{A}=0, \quad \bar{\kappa}_{n}^{B}=2, \quad \kappa_{g}^{A}=\frac{\bar{\kappa}_{n}^{B}}{\lambda}=-1, \quad \bar{\kappa}_{g}^{B}=\frac{\bar{\kappa}_{n}^{A}}{\lambda}=0, \quad \bar{\tau}_{g}^{A}=0, \bar{\tau}_{g}^{B}=0 .
$$

Example 2. Consider the parametric lightlike surface $S^{A}$ and the parametric spacelike surface $S^{B}$ as in the following

$$
\begin{aligned}
& \left.S^{A}: \quad \mathbf{A}\left(u_{1}, u_{2}\right)=\left(\sqrt{u_{1}^{2}+u_{2}^{2}}, u_{1}, u_{2}\right), \quad u_{1}, u_{2} \in\right]-5,5[, \\
& \left.S^{B}: \quad \mathbf{B}\left(v_{1}, v_{2}\right)=\left(-v_{2},-\cosh v_{1} \cos v_{2},-\cosh v_{1} \sin v_{2}\right), \quad v_{1}, v_{2} \in\right]-5,5[\text {. }
\end{aligned}
$$

Using (7.4), we obtain $\zeta^{A}=\frac{-1}{\sqrt{u_{1}^{2}+u_{2}^{2}}}\left(\sqrt{u_{1}^{2}+u_{2}^{2}}, u_{1}, u_{2}\right)$ and $\zeta^{B}=-\sinh \left(v_{1}\right)\left(\cosh v_{1},-\sin v_{2}, \cos v_{2}\right)$, therefore $\varepsilon^{A}=0 \forall\left(u_{1}, u_{2}\right) \in S^{A} ;\left(u_{1}, u_{2}\right) \neq(0,0)$ and $\varepsilon^{B}=-\sinh ^{4}\left(v_{1}\right) \forall\left(v_{1}, v_{2}\right) \in S^{B} ;\left(v_{1}, v_{2}\right) \neq$ $\left(0, v_{2}\right)$, thus the surfaces $S^{A}$ and $S^{B}$ are lightlike and spacelike, respectively. Considering the intersection point $P_{0}=\left(\frac{\pi}{2}, 0, \frac{\pi}{2}\right) \in S^{A} \cap S^{B}$ (see Figure 2), at this point, $\left(u_{1}, u_{2}\right)=\left(0, \frac{\pi}{2}\right),\left(v_{1}, v_{2}\right)=\left(-1.0232,-\frac{\pi}{2}\right)$ and $\lambda^{2}-\varepsilon^{A} \varepsilon^{B}=\frac{\pi^{2}\left(\pi^{2}-4\right)}{16} \neq 0$ (transversal intersection). Using (4.1), (4.3), (4.4), (4.7), (4.8), (5.1), (5.3), (5.5), and (7.4), we obtain

$$
\begin{gathered}
\mathbf{t}=(0.63662,-1,0.63662), \quad \boldsymbol{\alpha}^{\prime \prime}=(-0.258012,-0.405285,-0.894632), \\
\alpha^{\prime \prime \prime}=(0.313705,0.898052,0.313705)
\end{gathered}
$$

Thus the intersection curve at this point is a spacelike curve. Using (3.3), (3.4), (3.5), (3.6), (6.4) and (7.5), we obtain

$$
\begin{array}{cc}
\mathbf{n}=(-0.272264,-0.427671,-0.944047), & \kappa=0.947656 \\
\mathbf{b}=(1.21631,-0.427671,0.544527), & \tau=0.627667 .
\end{array}
$$




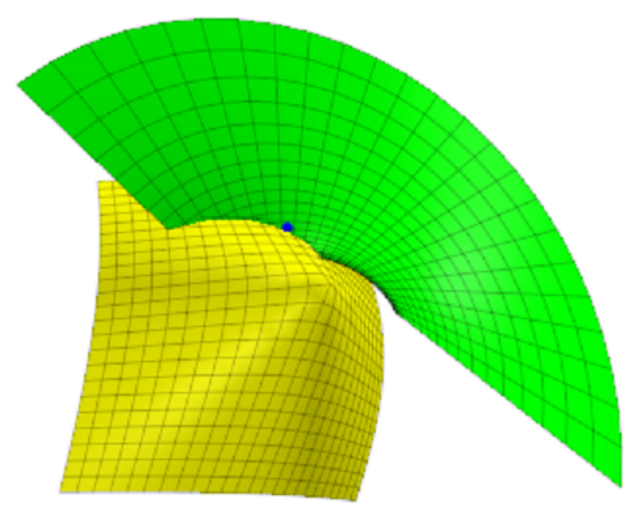

FIGURE 7.2. Transversal intersection of parametric lightlike and spacelike surfaces

Using (4.16), (4.18), (7.5) and (7.6), we obtain

$$
\mathbf{U}^{A}=(1.2974,-0.63662,0.29736), \quad \mathbf{U}^{B}=(-0.43384,-0.6815,0.63666)
$$

Using (4.17), (4.19), (6.3), (7.5), (7.6) and (7.7), we have the geodesic curvature, normal curvature and geodesic torsion as in the following

$$
\begin{array}{rlrl}
\left\|\zeta^{B}\right\| & =1.4674011, & \left\|\zeta^{B}\right\|^{2}=2.1532659, & \lambda=1.90281, \\
\bar{\kappa}_{n}^{A}=0.63662, & \bar{\tau}_{g}^{A}=0, & \kappa_{g}^{A}=\frac{\left\|\zeta^{B}\right\|^{2} \bar{\kappa}_{n}^{A}+2 \lambda\left\|\zeta^{B}\right\| \kappa_{n}^{B}}{2 \lambda^{2}}=0.705328, \\
\kappa_{n}^{B}=0.669138, & \tau_{g}^{B}=0.129093 & \kappa_{g}^{B}=\frac{\left\|\zeta^{B}\right\| \bar{\kappa}_{n}^{A}+\lambda \kappa_{n}^{B}}{|\lambda|}=1.16009 .
\end{array}
$$

Example 3. Consider the parametric lightlike surfaces $S^{A}$ and the implicit timelike surfaces $S^{B}$ as in the following

(7.8) $\quad S^{A}: \mathbf{A}\left(u_{1}, u_{2}\right)=\left(u_{1} \cosh u_{2}, u_{1} \sinh u_{2}, u_{1}\right) \quad S^{B}: \quad f\left(x_{1}, x_{2}, x_{3}\right)=-x_{1}+x_{2}+x_{3}=0$

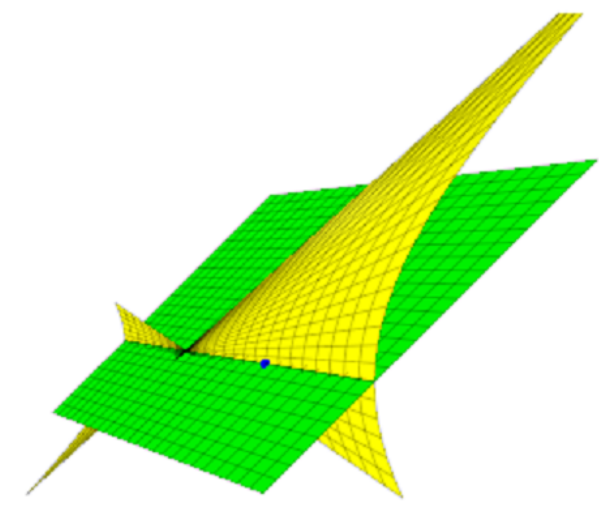

FIGURE 7.3. Transversal intersection of parametric lightlike and implicit timelike surfaces

Considering the intersection point $P_{0}=(1,0,1) \in S^{A} \cap S^{B}$ (see Figure 3). Using (7.8) at this point, we obtain $\zeta^{A}=\frac{1}{2}(1,0,1), \zeta^{B}=(1,1,1), \lambda=1 \neq 0, \varepsilon^{A}=0$ and $\bar{\varepsilon}^{B}=\varepsilon^{B}=1$, thus the surfaces $S^{A}$ and $S^{B}$ are lightlike and timelike, respectively. Using (4.9), (4.10), (4.13), (4.14) and (5.3) we obtain

$$
\alpha^{\prime}(s)=(1,0,1), \quad \alpha^{\prime \prime}(s)=(0,0,0),
$$


then the intersection curve is lightlike curve and it is a straight line.

Example 4. Consider the parametric timelike surface $S^{A}$ and the implicit lightlike surface $S^{B}$ as in the following

(7.9)

$$
S^{A}: A\left(u_{1}, u_{2}\right)=\left(\frac{u^{2}}{2}+u v+v, \frac{u^{2}}{2}+u v+v, u\right), \quad S^{B}: \quad f(x, y, z)=x+\arctan \left(\frac{z}{y}\right)=0 ; y^{2}+z^{2}<1
$$

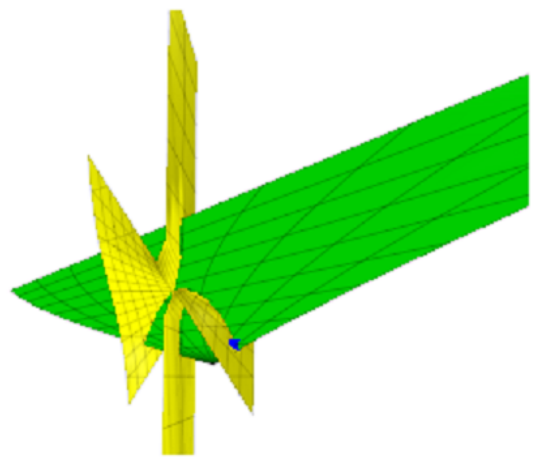

FIGURE 7.4. Transversal intersection of parametric timelile and implicit lightlike

From (7.9), we have $\zeta^{A}=(u+1, u+1,0)$ and $\zeta^{B}=\left(-1,-\frac{z}{y^{2}+z^{2}}, \frac{y}{y^{2}+z^{2}}\right)$, therefore $\varepsilon^{A}=0, \forall$ $\left(u_{1}, u_{2}\right) \in S^{A}$ and $\varepsilon^{B}>1, \forall y^{2}+z^{2}<1$, thus the surface $S^{A}$ is lightlike and the surface $S^{B}$ is timelike. Considering the point $P_{0}=\left(\frac{\pi}{8}, \frac{\pi}{8},-\frac{\pi}{8} \frac{\tan (\pi)}{8}\right) \in S^{A} \cap S^{B}$ (see Figure 4), in this point, we have $\lambda=1.59121, \varepsilon^{A}=0, \bar{\varepsilon}^{B}=1$, then the intersection curve is transversal. Using (4.10), (4.14), (5.1), (5.3), (5.5) and (7.9) we obtain

$$
\alpha^{\prime}=(-1.14379,-1.14379,1), \quad \alpha^{\prime \prime}=(4.0765,4.0765,0), \quad \alpha^{\prime \prime \prime}=(43.4607,43.4607,0)
$$

then the intersection curve at this point is a pseudo null curve. Using (3.9) , (3.11), (3.12) (6.6) and (7.10) we obtain

(7.11)

$$
\begin{aligned}
& \mathbf{t}=(-1.14379,-1.14379,1), \quad \mathbf{n}=(4.0765,4.0765,0), \quad \mathbf{b}=(-0.283116,-0.0378078,0.280581), \\
& \tau=-10.6613 .
\end{aligned}
$$

Using (4.18), (4.19), (6.5) and (7.11) we obtain

$$
\begin{gathered}
\bar{\kappa}_{n}^{A}=0, \quad \kappa_{n}^{B}=3.63771 \quad \bar{\tau}_{g}^{A}=0, \quad \bar{\tau}_{g}^{B}=3.83701, \\
\kappa_{g}^{A}=\frac{-\left\|\zeta^{B}\right\|^{2} \bar{\kappa}_{n}^{A}+2 \lambda\left\|\zeta^{B}\right\| \kappa_{n}^{B}}{2(\lambda)^{2}}=\frac{\left\|\zeta^{B}\right\| \kappa_{n}^{B}}{\lambda}=\frac{2.12953 \kappa_{n}^{B}}{1.59121}=4.8684, \\
\kappa_{g}^{B}=\frac{-\left\|\zeta^{B}\right\| \bar{\kappa}_{n}^{A}+\lambda \kappa_{n}^{B}}{|\lambda|}=\frac{\lambda \kappa_{n}^{B}}{|\lambda|}=\kappa_{n}^{B}=3.63771 .
\end{gathered}
$$

8. Conclusions. From the results obtained in this work we concluded that, we can computing the differential geometry properties of the transversal intersection curves of two surfaces, where each pair satisfies the following types spacelike-lightlike, timelike-lightlike and lightlike-lightlike using the information of surfaces involved. Moreover, the results obtained ends the study of differential geometry properties of the transversal intersection curves of two surfaces in Minkowski 3-space, because the cases spacelike-spacelike, timelike-timelike and timelike-spacelike were studied in [6], [22] and [16] respectively.

\section{REFERENCES}

[1] Abdel-All, N.H., Badr, S.A-N., Soliman, M.A., Hassan, S.A. Intersection curves of two implicit surfaces in $\mathbb{R}^{3}$. Journal of Mathematical and Computational Science, 2 (2), (2012), 152-171.

[2] Abdel-All, N. H., Badr, S. A-N., Soliman, M. A., Hassan, S. A. Intersection curves of hypersurfaces in $\mathbb{R}^{4}$. Computer Aided Geometric Design 29(2), (2012), 99-108. 
[3] Aléssio, O. Differential geometry of intersection curves of three implicit surfaces in $\mathbb{R}^{4}$. Computer Aided Geometric Design 26 (4) (2009) 455-471.

[4] Aléssio, O., Düldül, M., Düldül, B., Abdel-All, N. H., Badr, S. A.-N. Differential geometry of non-transversal intersection of three parametric hypersurfaces in Euclidean 4-space. Computer Aided Geometric Design 31(9), (2014), $712-727$.

[5] Aléssio, O., Düldül, M., Düldül, B., Abdel-All, N. H., Badr, S. A.-N. Differential geometry of non-transversal intersection curves of three implicit hypersurfaces in Euclidean 4-space. Journal of Computational and Applied Mathematics, 308 (2016) 20-38.

[6] Alessio, O., Guadalupe, I. V. Determination of a transversal intersection curve of two spacelike surfaces in Lorentz. Minkowski 3-space. Hadronic J. 30 (3) (2007) 315-341.

[7] Badr, S.A-N. Intrinsic geometry of intersection curves of surfaces in Euclidean spaces. Lambert Academic Publishing GmbH \& Co. KG, Germany, (2011).

[8] Badr, S. A.-N., Abdel-All, N. H., Aléssio, O., Düldül, M., Düldül, B. Non-transversal intersection curves of hypersurfaces in Euclidean 4-space. Journal of Computational and Applied Mathematics 288, (2015), 81-98.

[9] Bejancu, A. Lightlike curves in Lorentz manifolds. Publ. Math. Debrecen 44 (1994), 145-155.

[10] Bonnor, W. B. Null curves in a Minkowski space-time. Tensor N.S. 20 (1969), 229-242.

[11] Do Carmo, M.P. Differential geometry of curves and surfaces. Prentice-Hall, Inc., Englewood Cliffs, New Jersey, (1976).

[12] Duggal, K.L., Bejancu, A. Lightlike Submanifolds of semi-Riemannian manifolds and applications. Kluwer Academic Publishers, (1996)

[13] Duldül, B. U., Cąlışkan, M. Spacelike intersection curve of three spacelike hypersurfaces in $E_{1}^{4}$. Ann. Univ. Mariae CurieSkłodowska Sect. A 67 (1) (2013) 23-33.

[14] Goldman, R. Curvature formulas for implicit curves and surfaces. Comput. Aided Geom. Design 22 (7) (2005) $632-658$.

[15] Hassan, S.A., Badr, S.A-N. Differential geometry of self-intersection curves of a parametric surface in $\mathbb{R}^{3}$. Journal of Mathematical and Computational Science, 6 (6), (2016), 1108-1132.

[16] Karaametoglu, S., Aydemir, I. On the transversal intersection curve of spacelike and timelike surfaces in Minkowski 3-sapce. Journal of Science and Arts 16 (4) (2016) 345-356.

[17] Kuhnel, W. Differential geometry: curves-surfaces-manifolds. 4th Edition, Vieweg, Wiesbaden, 2008.

[18] Lopez, R. Differential geometry of curves and surfaces in Lorentz-Minkowski space. Int. Electron. J. Geom. 7 (1) (2014) $44-107$.

[19] O'Neill, B. Semi-Riemannian geometry with applications to relativity. Pure and Applied Mathematics, 103. Academic Press, Inc., New York, (1983).

[20] Ozdemir, M., Ergin, A. A. Spacelike Darboux curves in Minkowski 3-space. Differ. Geom. Dyn. Syst. 9 (2007) $131-137$.

[21] Ozdemir, M., Ergin, A. A. Non lightlike Darboux curves in Minkowski n-space. Hadronic J. Suppl. 19 (3) (2004) $343-351$.

[22] Sanh, Z., Yayh, Y. Non-null intersection curves of timelike surfaces in Lorentz-Minkowski 3-space. International Journal of Engineering and Applied Sciences 1 (3) (2014) 23-26.

[23] Soliman, M. A., Abdel-All, N. H., Hassan, S. A., Badr, S. A.-N. Intersection Curves of Implicit and Parametric Surfaces in $\mathbb{R}^{3}$. Applied Mathematics, 2 (8), (2011), 1019-1026.

[24] Topbas, E.S.Y., Gok, I., Ekmekci, N., Yayh, Y. Darboux frame of a curve lying on a lightlike surface. Mathematical Sciences and Applications E-Notes 4 (2) (2016) 121-130.

[25] Wang, Z., Pei, D. Null darboux developable and pseudo-spherical darboux iamge of null cartan curve in Minkowski 3-space. Hokkaido Mathematical Journal 40 (2011) 219-240.

[26] Ye, X., Maekawa, T. Differential geometry of intersection curves of two surfaces. Comput. Aided Geom. Design 16 (8) (1999) 767-788. 